Research Paper

\title{
Assessing debris flow impact on flexible ring net barrier: A coupled CFD-DEM study
}

\author{
Xingyue Li ${ }^{\text {a, }}$, Jidong Zhao ${ }^{\text {a,* }}$, Julian S.H. Kwan ${ }^{b}$ \\ ${ }^{a}$ Department of Civil and Environmental Engineering, Hong Kong University of Science and Technology, Hong Kong \\ ${ }^{\mathrm{b}}$ Geotechnical Engineering Office, Civil Engineering and Development Department, Hong Kong SAR Government, Hong Kong
}

\section{A R T I C L E I N F O}

\section{Keywords:}

Coupled CFD-DEM

Debris flow

Flexible ring net barrier

Multi-way interactions

\begin{abstract}
A B S T R A C T
Flexible ring net barriers have become increasingly popular in practical mitigation of debris flow worldwide. Systematic assessments of their response and performance subjected to the impact of realistic debris flows remain challenging. This study presents a novel computational approach based on coupled CFD-DEM to model the impact of debris flow on a flexible ring net barrier in a unified framework. The debris flow is treated as a solidfluid mixture, where the solid phase and the fluid phase are modeled by DEM and CFD, respectively. The barrier is simulated as a system of different deformable components, including rings, cables and energy dissipators, and is modeled with DEM. The proposed method expedites a convenient, unified consideration of multi-way interactions among the debris solid, the debris fluid, and the barrier. The simulation of a flexible barrier is calibrated against existing experimental data and past numerical results, by examining the quasi-static responses of different barrier components and the dynamic reactions of the entire barrier. The barrier system is further subjected to the impact of debris flows with different Froude numbers to examine its performance, in terms of its retaining capacity of debris mass and peak sustained forces in the barrier. Two energy-related indices, energy dissipation ratio and energy absorption ratio, are estimated for design reference. The study provides a novel, physically based predictive computational tool for future design and analysis of flexible ring net barriers in debris flow mitigation.
\end{abstract}

\section{Introduction}

Flexible barriers, in particular ring net barriers (see Fig. 1), have become a popular alternative to rigid structures in debris flow mitigation. Flexible barriers offer major advantages over conventional rigid resisting structures as being more cost and time effective in construction, convenient in transportation and installation in remote areas and less visual intrusion to surrounding landscape (Wendeler et al., 2018a,b). Key components of a typical flexible ring net barrier include interlocked rings, supporting cables, and energy dissipators (Wendeler et al., 2007; Albaba et al., 2017). The effectiveness of a flexible barrier to intercept a debris flow relies crucially on the cooperation of all its consisting components to dissipate the impact energy of the incoming debris flow, to bring down its mobility and to retain partially or wholly the solid mass. The process is commonly accompanied by large and frequently plastic deformations of the barrier components and intensive frictions and collisions among them (Castanon-Jano et al., 2017; Huo et al.,
2018). While it is feasible to perform pure structural analyses for a barrier system of this kind, e.g., by either experimental means or numerical tools such as finite element method (FEM) (Volkwein, 2004; Chan et al., 2012; Kwan et al., 2014), it poses tremendous challenges to consider its response and quantify its performance when subjected to the impact of a realistic debris flow. In FEM modeling of a flexible barrier impacted by debris flows, the debris impact has commonly been assumed to be a pseudo-static load onto the barrier (Wendeler et al., 2007; Kwan et al., 2014), which highly simplifies the dynamic impact process. Field tests on a natural gully or large-scale flume tests may yield more realistic results (Wendeler et al., 2008; Bugnion et al., 2012), but have various limitations related to the costs, scale and transferability of observations to different sites and cases. It is desirable to develop a general-purpose, cost-effective computational modeling and prediction tool that fully considers the physics and mechanics of the entire impact process of a debris flow onto a flexible barrier for practical analysis and design.

\footnotetext{
* Corresponding author.

E-mail address: jzhao@ust.hk (J. Zhao).

${ }^{1}$ Present address: Snow and Avalanche Simulation Laboratory, EPFL, Lausanne, Switzerland.
} 
The challenges pertaining to numerical modeling of debris flow impact on a flexible ring net barrier are threefold: (1) realistic modeling of debris flow. The kinematics and mobility of a debris low depend crucially on how its constituent phases interact with one another during its flow, which consequently affects the impacting process onto a barrier. Facing a complicated debris flow, simplications and hypotheses have been employed in many existing numerical studies, including the assumption of continuous solid and fluid phases and the neglection of variation along flow depth direction (Rhebergen et al., 2009; Dang and Meschke, 2014). It is important to recognize that debris flow can neither be treated as a flood or rock avalanche, but a typical two (or three) phase mixture of fluid and solid particles where intra-phase and inter-phase interactions dictate the flow behavior (Li and Zhao, 2018; Mao et al., 2020). (2) Robust simulation of flexible ring net barrier. As shown in Fig. 1, a typical flexible ring net barrier is comprised of several key components including rings, cables and energy dissipators which work collectively to resist the impact of debris flow. A proper design of such a barrier needs to rigorously consider intricate interactions between rings, between rings and cables and among rings, cables and energy dissipators. Noting the impact process is commonly brief but significant in terms of mass, force and momentum, the various interactions among the barrier components may commonly result in intense frictional sliding, rotating, stretching, bending of these components which may lead to excessive deformation and even failure of them. Force and moment sharing and transferring mechanisms among the different barrier components during the course of debris flow impact are difficult to be examined by existing experimental tools. However, they are critical to rational analyses of barrier performance for safe design. (3) Rational simulation of the impact process of a fluid-solid mixture onto a highly deformable flexible barrier. During the intense impacting process of a debris flow onto a flexible ring net barrier, highly complicated interaction processes between each phase of the debris mixture and all components of the flexible barriers occur, which is easily beyond any existing continnum modeling approaches can handle but serves critical physical bases for accurate quantification of key impact indices, such as peak impact forces and progressive retaining mass, to feed practical assessment and design of the barrier (Leonardi et al., 2016). Despite the wide use of flexible barriers in practical engineering problems, there lacks common design standard across the globe on flexible ring net barrier to mitigate debris flow impact.

In this study, we present a unified computational tool to model the multi-way interactions during the impact of debris flow onto a flexible ring net barrier, with a special focus to address the aforementioned challenges. A coupled Computational Fluid Dynamics and Discrete Element Method (CFD-DEM) approach is employed to simulate the debris flow as a solid-fluid mixture, and further use the DEM with bonded particles to model the entire flexible barrier system, including the rings, cables and energy dissipators. The DEM simulation of flexible ring net barrier enables us to capture the frictional sliding and collision among different barrier components and recover the well-known "curtain effect" (Chan et al., 2012; Albaba et al., 2017). The computational framework enables us to consider the solid-solid and solid-fluid interactions in a debris flow and the in-barrier component interactions of the ring net barrier (including ring-ring, cable-ring, ring-cable-energy dissipator interactions). It further empowers us to capture the debrisbarrier interactions during the impacting process, featured by the debris solid and barrier component interaction and debris fluid and barrier component interaction. Further interactions between both the debris flow and the barrier with the simulated channel bed can also be conveniently managed.

As will be demonstrated in the subsequent sections, the proposed approach offers us the possibility to quantitatively analyze various key aspects of barrier responses under debris impact that are necessary for design, including the barrier capacity in terms of blocked debris mass, the force distribution within the barrier and the force sustained in key barrier components. In addition, the energy dissipation and transfer can also be carefully examined with comprehensive consideration of the energy of incoming debris flow, the energy of outflowing debris flow, and the absorbed energy by a flexible barrier. In particular, in designing a multiple barrier system, debris energy dissipation and barrier interception efficiency are major indicators. Indeed, both quantities have been evaluated from the ratio of the absorbed energy by the barrier to the impact energy of the debris flow (Huo et al., 2017; Kwan et al., 2018; Song et al., 2019). However, the energy of outflow is rarely discussed

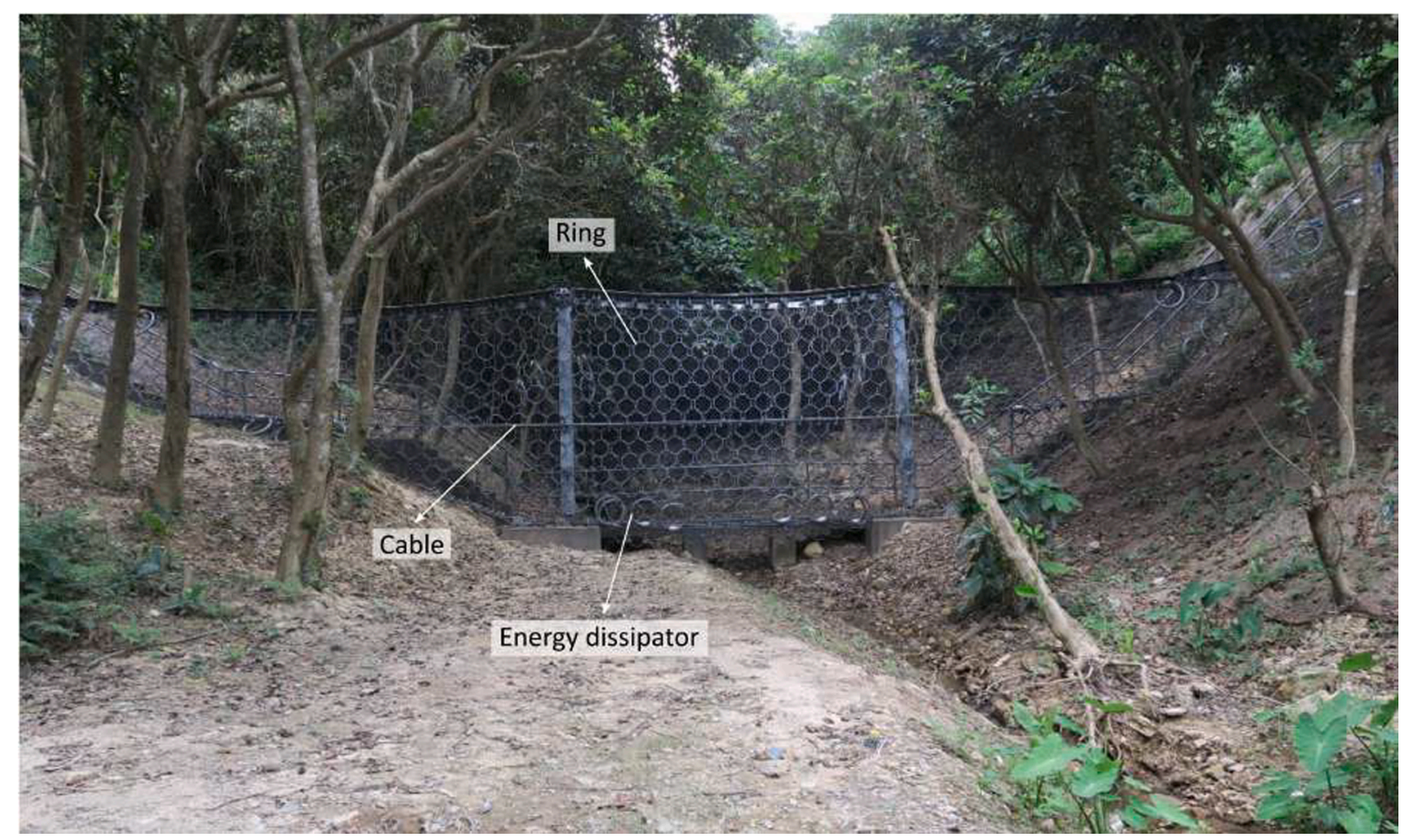

Fig. 1. A flexible ring net barrier for mitigation of debris flow in Hong Kong. 
but could be important for designing multiple barriers. These will all be discussed in this study in connection with the coupled CFD-DEM simulations.

It is noteworthy that the authors have previously used CFD-DEM approach to simulate single or double twisted wire net barriers under debris flow impact, where the wire net was simulated by DEM with particles connected by remote interaction (Li and Zhao, 2018). The wire units in this early study are bonded by nodes which do not allow the wire units undergo any frictional sliding or collisions. In contrast, each ring in a flexible ring net barrier to be treated here is a deformable unit that can interact with other components through frictional sliding and collisions, which is much more complicated and challenging to model. The impact by a multiphase debris flow further aggregates the complication of the simulation of a flexible ring net barrier. Moreover, twisted wire net barriers have been mainly applied in rockfall drapery systems (Giacomini et al., 2012; Marchelli et al., 2019) and low-energy rockfall barriers (Buzzi et al., 2013; Gao et al., 2018) and are practically less popular in large scale debris flow prevention projects, since their retention/energy capacity is relatively low. In contrast, flexible ring net barriers can sustain much larger impact load and retain more debris mass, which have been widely used worldwide (Wendeler et al., 2018a; Volkwein et al., 2015). Therefore, with a focus on flexible ring net barrier subjected to the impact of multiphase debris flow, the present study is both technically challenging and practically significant.

\section{Methodology and formulation}

\subsection{Flexible ring net barrier}

A flexible barrier allows debris fluid and fine debris particles pass through in the impacting process, a key feature distinguishing it from the more conventional rigid barrier. Its permeability, however, may decrease with the gradual retainment of debris solids inside the barrier. Therefore, it is vital to simulate a debris flow as a mixture of viscous fluid and particles for twofold purposes: (1) It helps capture the particlefluid interactions within a debris flow that underpin its dynamics; (2) It enables us to capture the realistic behavior of fluid phase passing through the barrier while solid phase is intercepted. In this study, we consider a natural debris flow as a mixture of fluid and solid particles. The CFD is employed to solve the locally averaged Navier-Stokes equation for the fluid phase, and the DEM is used to solve the Newton's equations for the particle system. The fluid-particle interactions are considered by exchanging interaction forces between the CFD and DEM computations, including buoyancy and drag force (Goniva et al., 2010; Zhao and Shan, 2013; Shan and Zhao, 2014; Li and Zhaoi, 2018). To simulate a flexible ring net barrier in a unified computational framework, the DEM is exploited for the modeling of the barrier. In so doing, the multi-way interactions among the debris fluid, the debris solid, and the barrier can be seamlessly captured with the coupled CFDDEM method. Detail of the CFD-DEM approach can be found in Zhao and Shan (2013), Shan and Zhao (2014), Tao and Tao (2017), Li and Zhao (2018) and will not be repeated here. Emphasis of this study is placed on the incorporation of simulation of flexible ring net barrier within the (a)

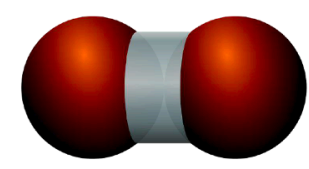

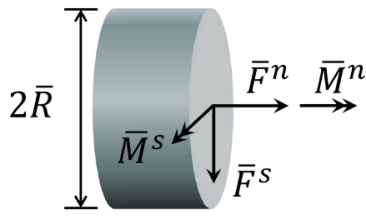

(b)

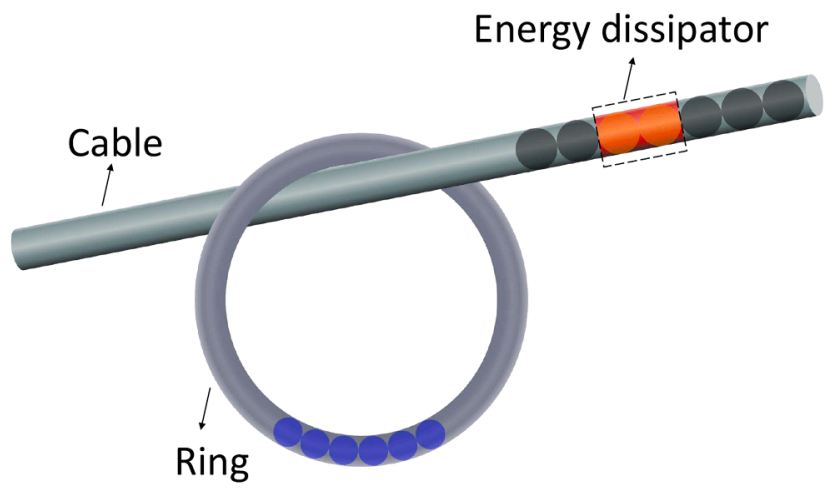

(c)

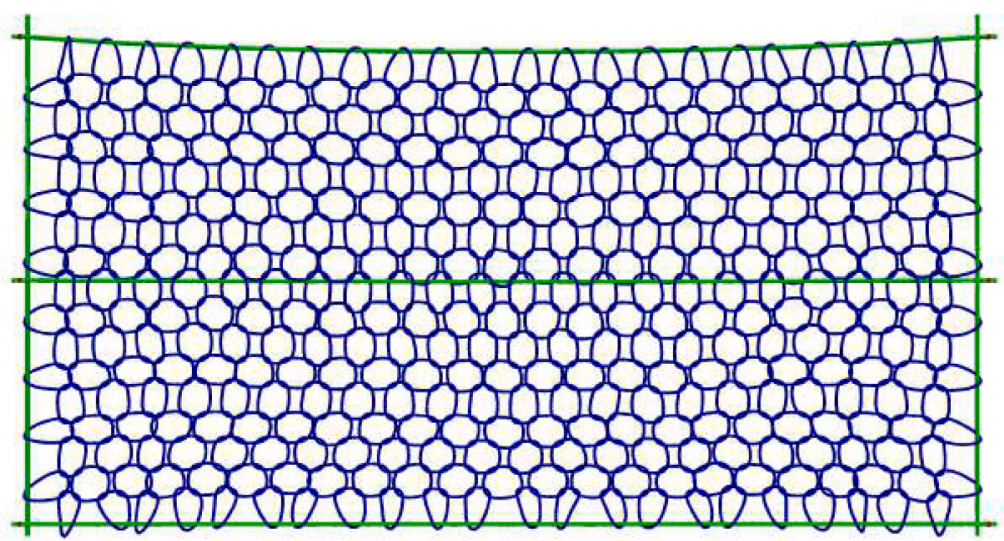

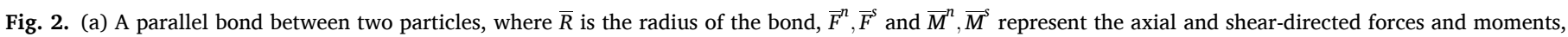

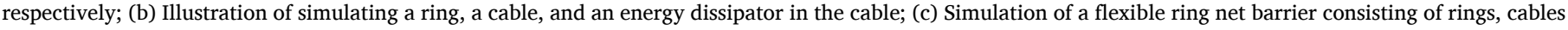
and energy dissipators. 
same coupled CFD-DEM framework.

As mentioned in the introduction, we have modeled single or double twisted wire net type flexible barriers using DEM with particles connected by remote interaction (Li and Zhao, 2018), which is relatively less challenging as the individual wire units are inter-bonded with their neighbouring units by wires without allowing relative motions (unless broken). However, in a flexible ring net barrier, each ring is a deformable unit that interacts with its neighbouring rings through inter-ring frictional sliding, collision and stretching. To address this challenge, we borrow the concept for modeling rockfall protection fence (Nicot et al., 2001; Bertrand et al., 2008; Thoeni et al., 2014) and the bondedparticle model (Potyondy and Cundall, 2004), and simulate all the barrier components by bonded particles as illustrated in Fig. 2. Specifically, we adopt particles with different bonds to capture distinct behaviors of rings, cables and energy dissipators. To simulate each of the barrier components, three computational steps are followed.

(1) Determination of particle location and particle properties. Based on the geometry of a barrier component, particles can be so arranged to form the needed shape. Take the ring in Fig. 2b as an

(a)

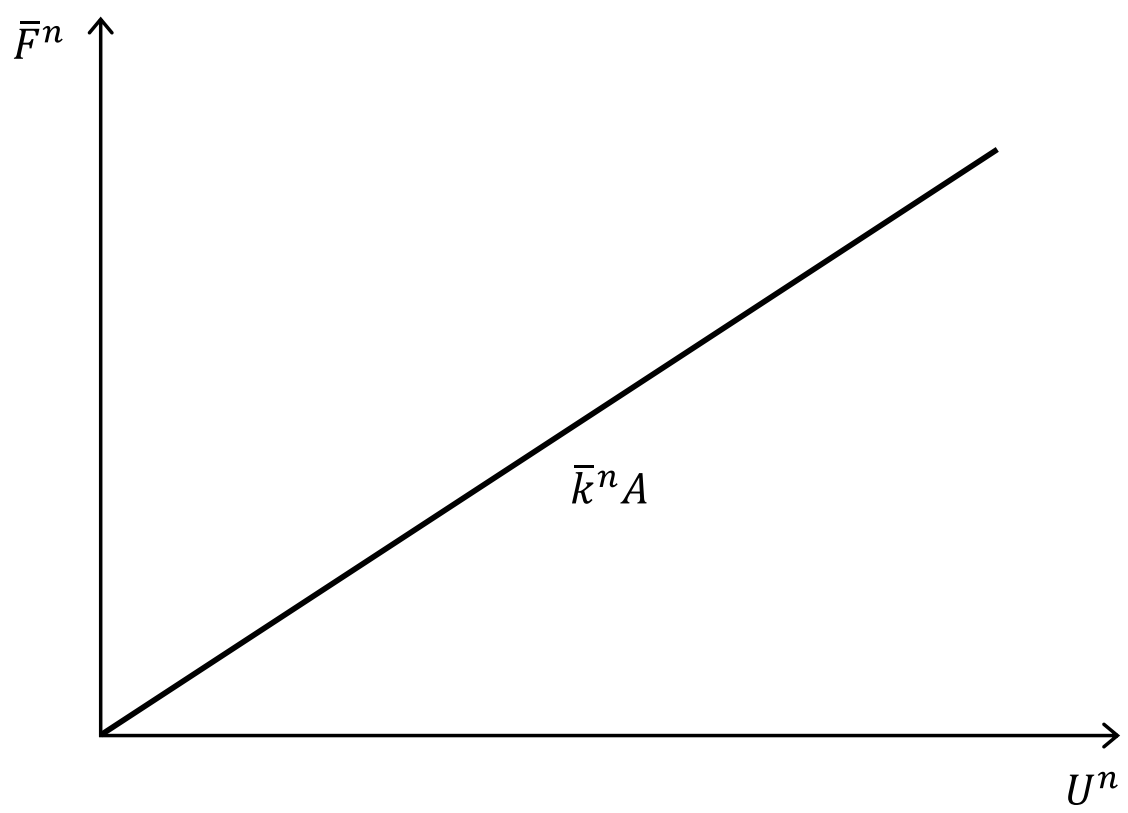

(b)

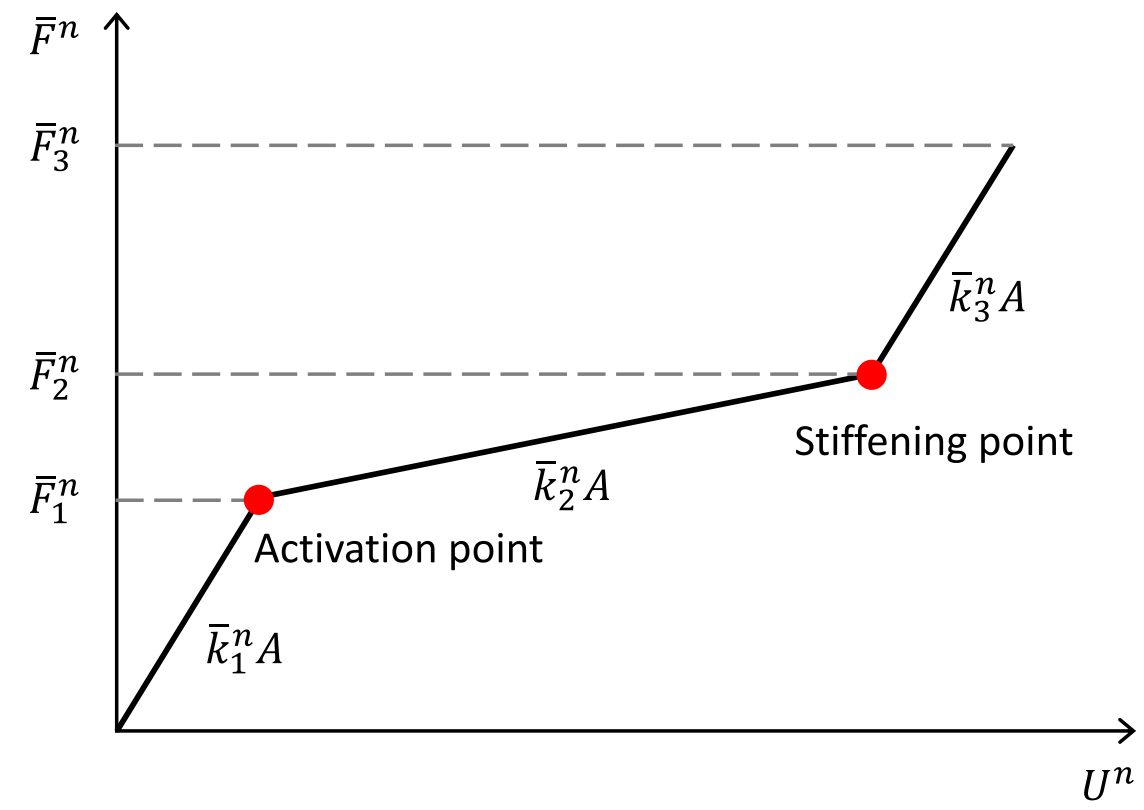

Fig. 3. (a) A linear relation between axial force and axial deformation of a parallel bond, $\bar{k}^{n}$ is the normal stiffness per unit area, $A$ is the area of the cross section of the bond; (b) A tri-linear law of a parallel bond for modeling the behavior of an energy dissipator (modified based on Castanon-Jano et al. (2017)), $\bar{k}_{1}^{n}, \bar{k}_{2}^{n}, \bar{k}_{3}^{n}$ are the normal stiffness per unit area at the three stages, respectively. 
example, a group of particles is placed along the centerline of the ring. The diameters of all these particles are assumed to be identical and are estimated according to the thickness of the ring. As the particles are in contact with one another, a thin ring may need a great number of particles which cause high computational cost. Under this condition, the particle size may need to be adjusted. After deciding the particle size, the density of particles can be calculated by placing a constraint that the total mass of all particles is equal to that of the ring. Similar procedures can be carried out for the simulation of the cable, where the particles are located along a straight line. Modeling the shapes of rings and cables can recover frictional sliding among the rings and between the rings and cables, which may help to redistribute the load within a barrier in a physically reasonable manner. Regarding the energy dissipator, we can simply capture its function without actual consideration of its original shape. As demonstrated in Fig. 2b, two bonded particles are used to simulate the energy dissipator embedded in the cable. It is the bond between the two particles, instead of the particles themselves, that governs the mechanical response of the simulated energy dissipator.

(2) Implementation of parallel bonds. To form a barrier component with the particles in Step (1), virtual parallel bonds are applied to connect the particles. Different from contact bonds which can only sustain forces, parallel bonds can sustain both forces and moments as shown in Fig. 2a. Detail of calculating the forces and moments can be found in Potyondy and Cundall (2004) and Li and Zhao (2018). In this study, the normal stiffness of parallel bonds for the modeling of rings and cables is assumed to be constant, by considering a linear relation between the axial force $\bar{F}^{n}$ and axial deformation $U^{n}$ of the bonds as shown in Fig. 3a. To capture the behavior of an energy dissipator, a trilinear law is further implemented as illustrated in Fig. 3b. In practice, energy dissipators are designed to absorb energy from the impacting debris mass. Different types of energy dissipators may function differently in term of dissipation mechanisms, ranging from plastic deformation, heat generated by friction, and partial failure like fracture and breakage (Castanon-Jano et al., 2017). Depending on the design and configuration of the energy dissipators, their force-displacement response can vary and be highly non-linear in reality. In typical quasi-static load test, many energy dissipators show a tri-linear load-deformation relationship. The tri-linear law is adopted in this study to describe the typical response of an energy dissipator that dissipates energy by both friction and plastic deformation. As shown in Fig. 3b, the normal stiffness of the bond is varied at the three stages.

$$
\bar{k}^{n}= \begin{cases}\bar{k}_{1}^{n}, & \bar{F}^{n}<\bar{F}_{1}^{n} \\ \bar{k}_{2}^{n}, & \bar{F}_{1}^{n} \leqslant \bar{F}^{n}<\bar{F}_{2}^{n} \\ \bar{k}_{3}^{n}, & \bar{F}_{2}^{n} \leqslant \bar{F}^{n}<\bar{F}_{3}^{n}\end{cases}
$$

The first stage captures the elastic deformation of the energy dissipator when its axial force $\bar{F}^{n}$ is smaller than the activation force $\bar{F}_{1}^{n}$. After the axial force $\bar{F}^{n}$ reaches the activation force $\bar{F}_{1}^{n}$, the second stage models the plastic deformation of the energy dissipator, at which the most energy is dissipated. The stiffness of the energy dissipator at this stage is normally smaller than that at the first stage. Once the axial force reaches $\bar{F}_{2}^{n}$, the energy dissipator is stiffened and it behaves as a cable (Castanon-Jano et al., 2017). The tri-linear model can be abridged to obtain a bi-linear model if needed, by assigning an extremely large value to $\bar{F}_{2}^{n}$ so that the third stage is never reached.

Having the forces and moments on a parallel bond, the maximum tensile and shear stresses acting on the parallel-bond periphery can be obtained from the beam theory in structural mechanics. If the maximum tensile stress exceeds the tensile strength of the bond or the maximum shear stress exceeds the shear strength of the bond, the parallel bond will break. This breakage criterion of a parallel bond follows that in Potyondy and Cundall (2004).

(3) Calibration of model parameters. Two groups of parameters, related to the particles and the parallel bonds, need to be carefully calibrated and then applied in the simulation. In modeling debris flow impact on a flexible barrier, the parameters of the barrier particles control the physical interactions on the barrier from the debris flow, including the friction and collision from the debris particles as well as the buoyancy and drag force from the debris fluid. The properties of the parallel bonds govern the response of the barrier to the physical interactions, such as deformation of the barrier and load transfer/distribution within the barrier.

By simulating a flexible ring net barrier with the DEM, key geometrical and mechanical characteristics of the barrier components can be faithfully captured. The shapes of rings and cables can be reproduced with bonded particles, which facilitate the modeling of sliding, twisting and collision between the rings and the cables. The elastic-plastic-stiffening behavior of an energy dissipator can be reproduced with the implementation of a tri-linear law. Note that the contact force arising from particle-particle overlap is not affected by the bond model. If two particles are connected by a bond and meanwhile have overlap, both the bond force and the contact force apply. When the bond breaks, the bond force will cease to apply while the contact force will remain if there is still interparticle overlap.

\subsection{Multiple-way interactions in debris flow impacting on a flexible ring net barrier}

The above DEM simulation of flexible ring net barrier can be readily integrated into the coupled CFD-DEM framework described in Zhao and Shan (2013), Shan and Zhao (2014) and Li and Zhaoi (2018). The same DEM solution procedure can be applied to the modeling of debris solid particles and the flexible ring net barrier, albeit using different contact or bond models. The interaction between the flexible ring net barrier and the debris particles can be readily executed according to the same contact laws for debris particles. In addition, channel walls can also be modeled by DEM in the framework. As such, a single DEM framework is capable of simulating the following different interactions during a typical debris flow impact: (a) between debris particles; (b) between a debris particle and a flexible barrier component (ring, cable or energy dissipator); (c) between any two components of the flexible ring net barrier (e.g., ring-ring and ring-cable); (d) between a debris particle and a channel wall. Meanwhile, the debris fluid is modeled by the CFD, and the CFD exchanges interaction forces with DEM to compute the following fluid-solid interacting processes during a typical debris flow impact on a flexible ring net barrier: (a) between debris fluid and debris particles; (b) between debris fluid and any component of the flexible barrier; (c) between debris fluid and a channel wall. Consequently, the multi-way interactions among the flexible barrier, the debris particles and the debris fluid can be seamlessly considered within the unified CFD-DEM framework.

\section{Calibrations and benchmarking}

The coupled CFD-DEM method has been benchmarked and verified with classical geomechanics problems (Zhao and Shan, 2013; Shan and Zhao, 2014), including one-dimensional consolidation, single particle settling from air to water, and dynamic impact of granular flow on water reservoir. In addition, its capability in modeling twisted wire net barriers has been verified in Li and Zhao (2018). To further benchmark the proposed CFD-DEM for studying debris flow impacts on a flexible ring net barrier, quasi-static loading on the barrier components (i.e., ring and 
energy dissipator) and dynamic loading of rockfall on an entire barrier are simulated. Particular interests are placed on the force-displacement responses of the ring and the energy dissipator under the quasi-static loading. Both displacement and energy of the rockfall are investigated during its dynamic impact on the barrier. All the simulation results will be quantitatively compared with the collected experimental and numerical data from literature.

\subsection{Single ring}

Fig. 4a depicts the model setup of the quasi-static loading on a single ring, referring to the experiment by Volkwein (2004). The left end of the ring is fixed by the particle in blue. Its right end is pulled by applying an external force $F$ to the red particle. Our DEM simulation of the ring uses 60 particles connected with 60 parallel bonds, which are found sufficient to capture the behavior of the ring with affordable computational cost based on sensitivity studies. Adopted parameters for the particles are summarized in Table 1 . The particle diameter is determined based on the size of the ring in the experiment (Volkwein, 2004), by dividing the perimeter of the ring $(942.5 \mathrm{~mm})$ with the number of particles $(60)$. As mentioned in Section 2, the response of the ring (i.e., deformation and load transfer) is mainly controlled by the bond properties. Hence, the inter-particle friction coefficient is set to zero to minimize the contact effect of the particles on the deformation of the ring under the external

(a)

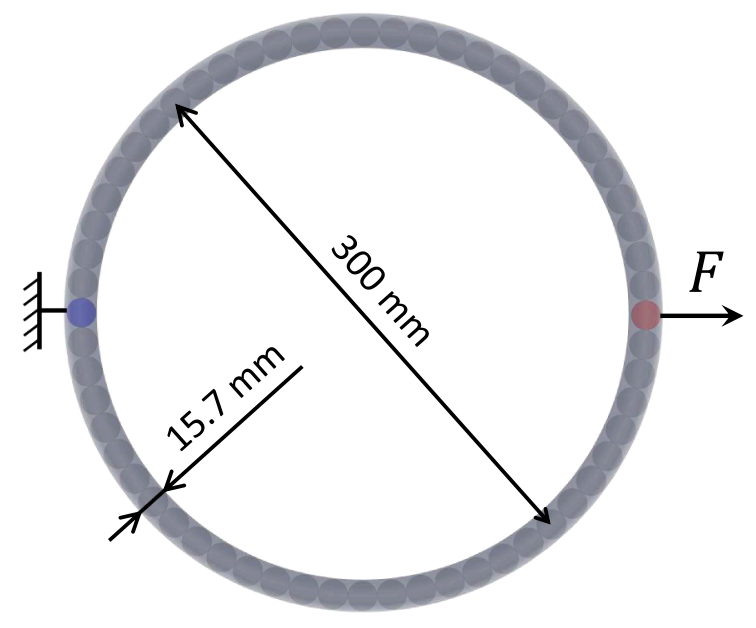

(b)

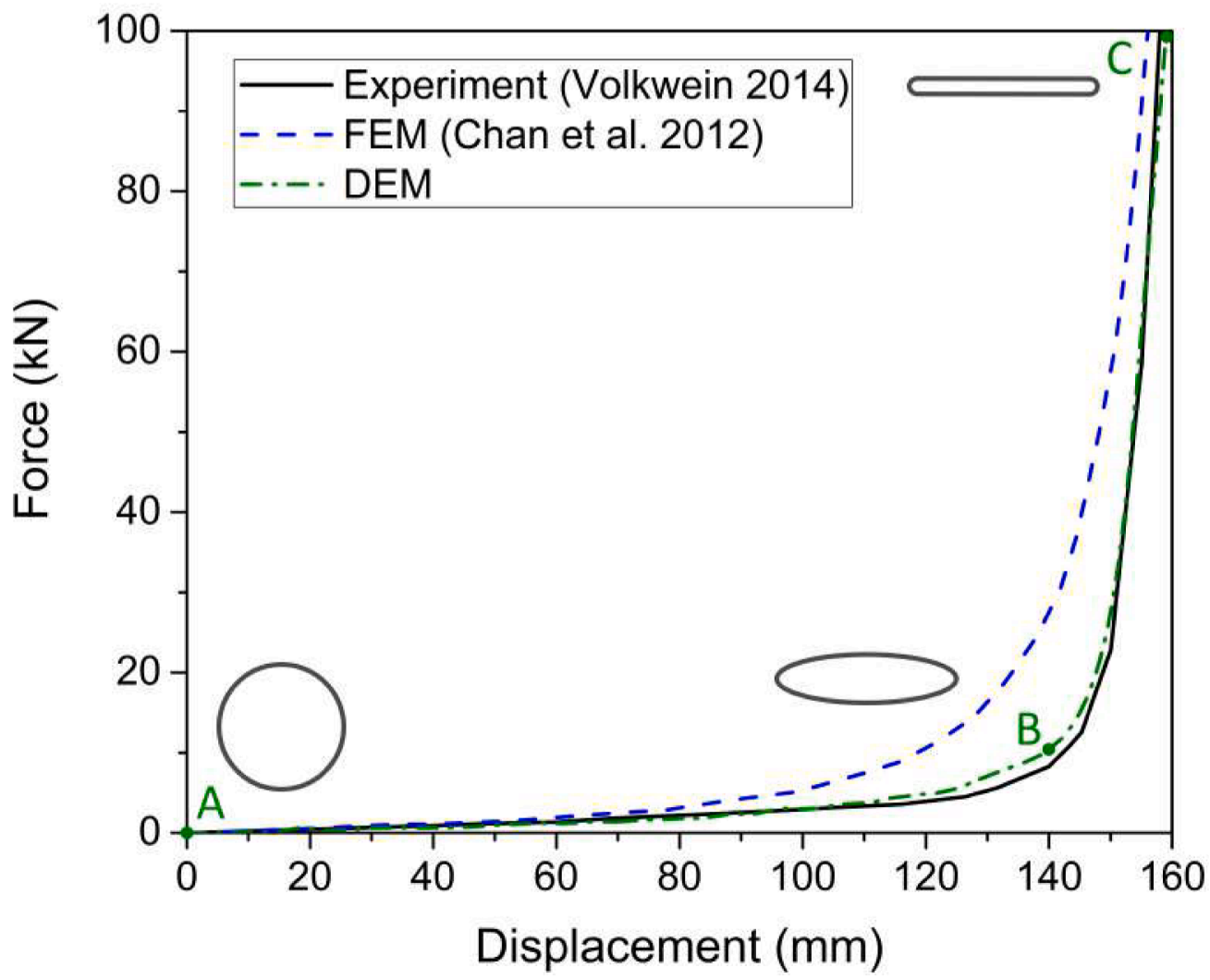

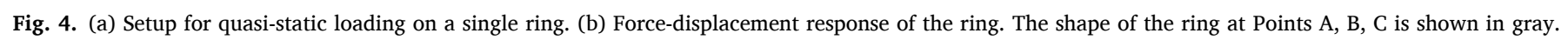


Table 1

Adopted parameters for particles of the ring in the quasi-static loading simulation.

\begin{tabular}{lllll}
\hline Diameter & Density & $\begin{array}{l}\text { Young's } \\
\text { modulus }\end{array}$ & $\begin{array}{l}\text { Poisson's } \\
\text { ratio }\end{array}$ & $\begin{array}{l}\text { Friction } \\
\text { coefficient }\end{array}$ \\
\hline $15.7 \mathrm{~mm}$ & $\begin{array}{l}1000 \mathrm{~kg} / \\
\mathrm{m}^{3}\end{array}$ & $5 \mathrm{MPa}$ & 0.3 & 0.0 \\
\hline
\end{tabular}

loading. Note this zero friction coefficient is only applicable for particleparticle contacts within the same ring. In the presence of multiple rings and other objects, the friction between rings and between rings and other objects should be assigned according to that measured in experiments or calibrated in past numerical modeling, such as in the rockfall impact case in Section 3.3.

Fig. 4b shows the obtained relation between the external force and the displacement from the DEM simulation, in comparison with that from the experiment by Volkwein (2004) and the Finite Element Modeling (FEM) by Chan et al. (2012). The displacement is calculated based on the right point of the ring (in red in Fig. 4a) where the external force is acted on. All three curves in Fig. 4b can be largely divided into two stages according to the growth rate of force. At the first stage where the displacement is approximately smaller than $140 \mathrm{~mm}$, the shape of the ring changes significantly from an initially perfect circle to an ellipse-like shape with a high aspect ratio (from point A to point B in Fig. 4b). Since the perimeter of the ring does not vary significantly at this stage, the force increases gently with the displacement. At the second stage, the perimeter of the ring begins to show a notable increase, resulting in a sharp growth of force. The physics of these two stages indicate that the shear stiffness and the normal stiffness of the parallel bonds control the behavior of the ring at the first and the second stages, respectively. Using low shear stiffness $\left(8 \times 10^{8} \mathrm{~N} / \mathrm{m}\right)$ and high normal stiffness $\left(1.3 \times 10^{12} \mathrm{~N} / \mathrm{m}\right)$ for the parallel bonds, the DEM simulation satisfactorily recovers the response of the ring as observed in the experiment. Compared to the FEM data, the DEM prediction may render a better consistency with the experimental result. One attributable reason may be the use of quadrate element in the FEM modeling for the ring in Chan et al. (2012) which appears to be oversimplified. As the real shape of the ring is considered in the DEM simulation, both the bending and axial behaviors of the ring at the first and second stages in Fig. 5 are reasonably captured.

\subsection{Energy dissipator in a cable}

As cables are the key to transfer the impact load of debris flow to lateral fixed anchors of a flexible barrier, energy dissipators are commonly embedded into a cable to dissipate energy. Among different types of energy dissipators (Castanon-Jano et al., 2017), the most

(a)

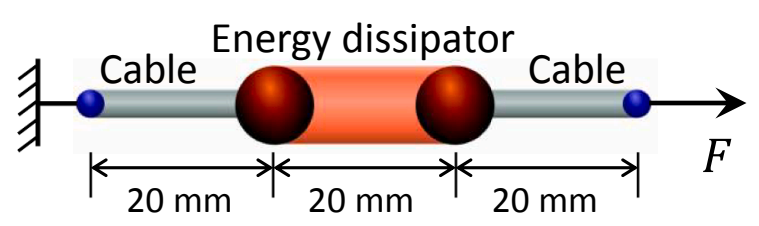

(b)

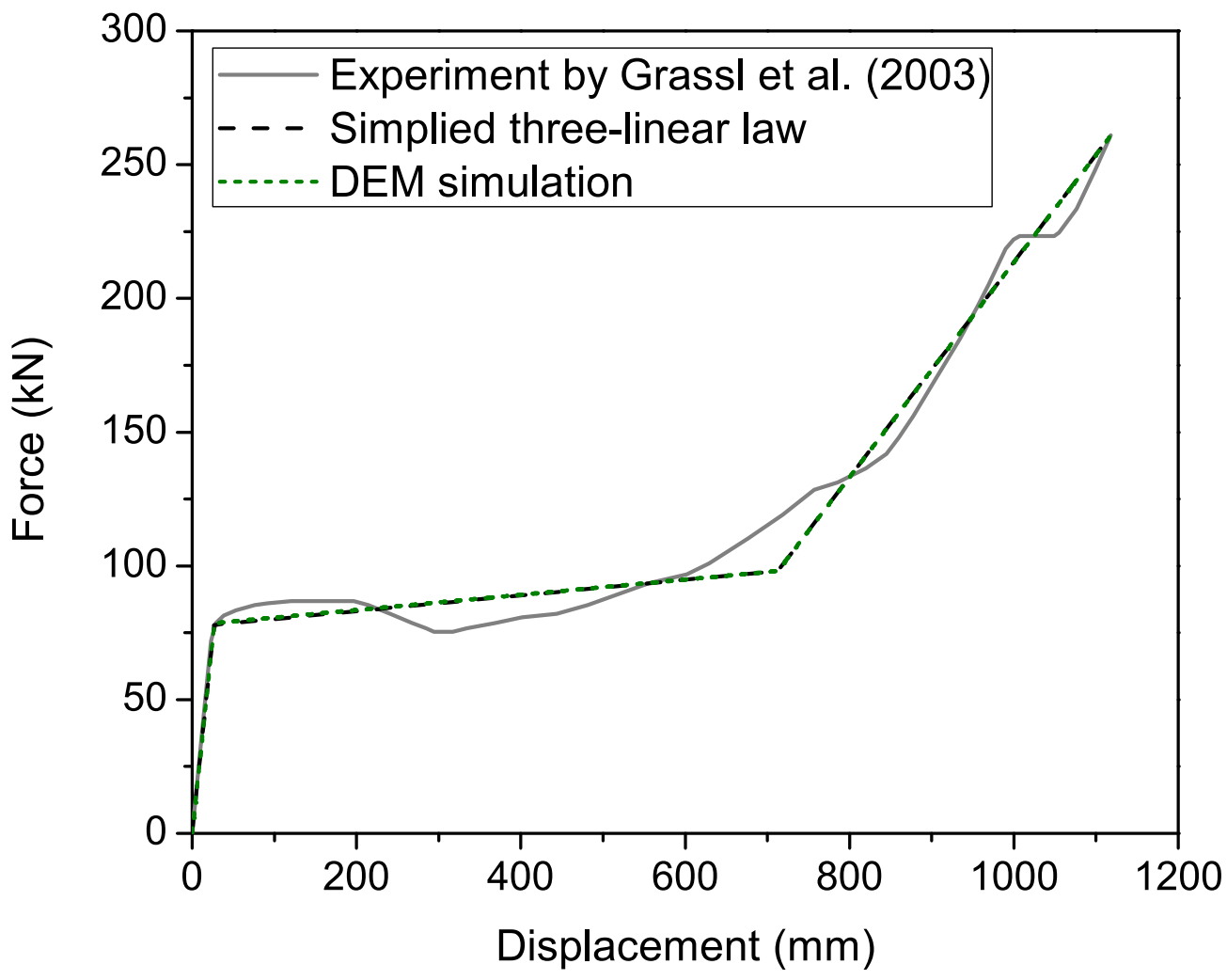

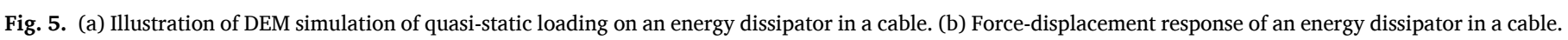


popular one in debris flow mitigation is dissipating energy by both friction and plastic deformation, which is the focus here. Fig. 5a demonstrates the numerical setup of quasi-static loading on an energy dissipator in a cable, according to the experiment by Grassl et al. (2003). The energy dissipator is modeled by two particles connected with a parallel bond (in red in Fig. 5a). These two particles are further connected to the cable particles (in blue) by two parallel bonds (in gray) at its two ends. The left end of the cable is fixed and the right end sustains an external force $F$. As the energy dissipator and cable are under tension, physical contact between particles will not occur. Consequently, properties of the simulated particles have negligible effect on the simulation result for this example. In addition, the shear stiffness of the bonds plays a trivial role too, which is assumed to be identical to the normal stiffness of the bonds. To minimize the variables to be calibrated, the stiffness of the cable is assumed to be the same as the stiffness of the energy dissipator at the elastic stage.

The force-displacement response of the energy dissipator in the experiment is highly non-linear, as shown in Fig. 5b. Various simplified models have been employed to capture a similar amount of energy dissipation (i.e., the area below the curve), including bi-linear laws and tri-linear laws (Castanon-Jano et al., 2017). As illustrated in Fig. 5b, we interpret the non-linear relation to a simplified tri-linear law (dash line) defined in Section 2, which is to be duplicated by our DEM simulation. In the DEM simulation, the force-displacement response is primarily controlled by the normal stiffness of the energy dissipator. Using the stiffnesses and force criteria listed in Table 2, the tri-linear behavior is perfectly reproduced with the DEM simulation (short-dash line in Fig. 5b). The adopted parameters in Table 2 agree with the discussion of Fig. 3. The bond stiffness in the second stage $\bar{k}_{2}^{n}$ is smaller than that during the first stage $\bar{k}_{1}^{n}$, which leads to a longer displacement and higher dissipated energy over the second stage in Fig. 5b. The growth of the bond stiffness at the third stage $\bar{k}_{3}^{n}$ reflects the stiffening of the energy dissipator. Note that, in reality, the distinct responses of the energy dissipator at the three stages are due to elastic deformation, plastic deformation and stiffening, respectively. The DEM approach in this study simulates all the barrier components as elastic elements, which is an approximation. Nevertheless, with proper calibration of the model parameters, large deformation and changing stiffness of the barrier component can indeed be captured.

\subsection{Rockfall impact on a flexible ring net barrier}

Referring to the experiment by Volkwein (2004), the setup in Fig. 6a is used in our DEM modeling to examine the scenario of rockfall impact on a flexible ring net barrier, as an analogy to debris particles impacting onto a flexible barrier. The rockfall is modeled as a spherical ball with a diameter of $0.82 \mathrm{~m}$ and a mass of $825 \mathrm{~kg}$, which is initially located $16 \mathrm{~m}$ above the ring net before being released to fall to the center of the ring net under gravity. Identical rings with a diameter of $300 \mathrm{~mm}$ are used to form the quadrate ring net $(3.9 \mathrm{~m}$ by $3.9 \mathrm{~m})$. The boundary rings at the four edges of the net are attached to four cables, respectively. The motion of the cables is restrained at the four corners of the ring net by using fixed bars and constrainers, with which the cables can freely slide and deform along the axial direction of the cables but not along the radial direction. Energy dissipators are placed close to the ends of the cables, which are expected to be activated according to the experimental

Table 2

Properties of the parallel bond forming the energy dissipator in the DEM quasistatic loading simulation.

\begin{tabular}{lllllll}
\hline \multicolumn{3}{c}{ Bond stiffness (N/m) } & & & \multicolumn{3}{c}{ Bond force (kN) } \\
\cline { 1 - 3 } $\bar{k}_{1}^{n}$ & $\bar{k}_{2}^{n}$ & $\bar{k}_{3}^{n}$ & & $\bar{F}_{1}^{n}$ & $\bar{F}_{2}^{n}$ & $\bar{F}_{3}^{n}$ \\
\hline $3.7 \times 10^{10}$ & $1.2 \times 10^{8}$ & $1.9 \times 10^{9}$ & & 78.0 & 98.2 & 261.0 \\
\hline
\end{tabular}

observation (Volkwein, 2004). In the DEM simulation, the particles at the ends of the cables are fixed, replicating the anchored ends of the cables in the experiment (Volkwein, 2004).

The particle properties adopted in the simulation are summarized in Table 3, which are partially extracted from the FEM simulation of the experiment (Koo et al., 2017), including the density and friction of the spherical ball, the ring and the cable. The properties related to the deformation of the ring and the cable, such as Young's modulus and Poisson's ratio, cannot be directly borrowed from the FEM modeling (Koo et al., 2017), since the deformation response in our DEM simulation is primarily controlled by the properties of parallel bonds. Note that the ball in the rockfall test by Volkwein (2004) was made of steel fiber reinforced concrete (Volkwein, 2004; Yiu et al., 2012), the adopted Young's modulus and Poisson's ratio for the spherical ball in Table 3 refer to the experimental measurements of steel fiber reinforced concrete (Lau and Anson, 2006). For simplification, the shear stiffness of all the parallel bonds comprising the barrier is assumed to be identical to the normal stiffness of the bonds. A bi-linear force-displacement relation is adopted for the bonds of the energy dissipators. Accordingly, two stiffnesses $\left(3.5 \times 10^{8} \mathrm{~N} / \mathrm{m}\right.$ and $3.5 \times 10^{7} \mathrm{~N} / \mathrm{m}$ in Table 3$)$ are used before and after the normal force of the bond reaches the activation force. The bi-linear model is abridged from the tri-linear model, by setting an extremely large value to the force at the stiffening point in Fig. $3 \mathrm{~b}\left(1 \times 10^{50} \mathrm{~N}\right.$ in this study).

Under gravity, the ring net which is initially horizontal shows a sagging behavior before the impact of the ball (Fig. 6a). After the ball plummets into the net, the rings quickly rearrange themselves to sustain the impact load. The boundary rings attached to the cables slide along and drag down the cables as demonstrated in Fig. 6b. The sliding of the ring net along the surrounding cables is called the curtain effect in the literature (Volkwein, 2005; Bertrand et al., 2012; Coulibaly et al., 2019). Moreover, notable elongation of the energy dissipators is caught, consistent with the experimental observation. The displacement and energy of the ball are examined as shown in Fig. 7, by comparing the DEM simulation results with the experimental data by Volkwein (2004). Time zero corresponds to the instant that the ball reaches the initial horizontal plane of the ring net. The displacement of the ball is calculated from time zero, with a positive direction pointing upwards. It is straightforward in Fig. 7a that the initial decrease and later increase of the displacement match the falling down and bouncing up processes of the ball, respectively. The energy of the ball in Fig. $7 \mathrm{~b}$ consists of potential energy and kinetic energy. At the very beginning $(0 \mathrm{~s}<\mathrm{t}<0.05$ $\mathrm{s})$, the ball arrives at the position of the ring net, with an ever increasing velocity. Reasonably, the energy of the ball does not show notable reduction, as a result of the increasing kinetic energy and decreasing potential energy. The nonlinear decrease of energy partly reflects the energy loss due to dissipations overcoming its frictional contacts with the ring net and further cross the entire system. As the impeding effect of the ring net becomes notable, the kinetic energy of the ball starts to reduce along with the reduction of its potential energy, leading to a drop of total energy from $t=0.05 \mathrm{~s}$ to $t=0.25 \mathrm{~s}$. The instant which the lowest energy is reached in Fig. $7 \mathrm{~b}$ coincides well with that of the smallest displacement in Fig. 7b, at which the velocity of the ball vanishes. After the ball begins to bounce up from $t=0.25 \mathrm{~s}$, its total energy show a sensible increase. As evidenced in Fig. 7a, the displacement obtained from the DEM modeling agrees well with the experimental record. The energy of the ball from the DEM and the experiment in Fig. 7b also show reasonable consistency. Note that compared with the prestressed cables in the experiment by Volkwein (2004), the cables in the DEM modeling are not preloaded, which may contribute to the discrepancy observed at the beginning of the energy evolution $(0.05 \mathrm{~s}<\mathrm{t}<0.15 \mathrm{~s})$. Nevertheless, the above example clearly shows how complicated, nonlinear interactions among different structural components can become even with such a simple model case. 


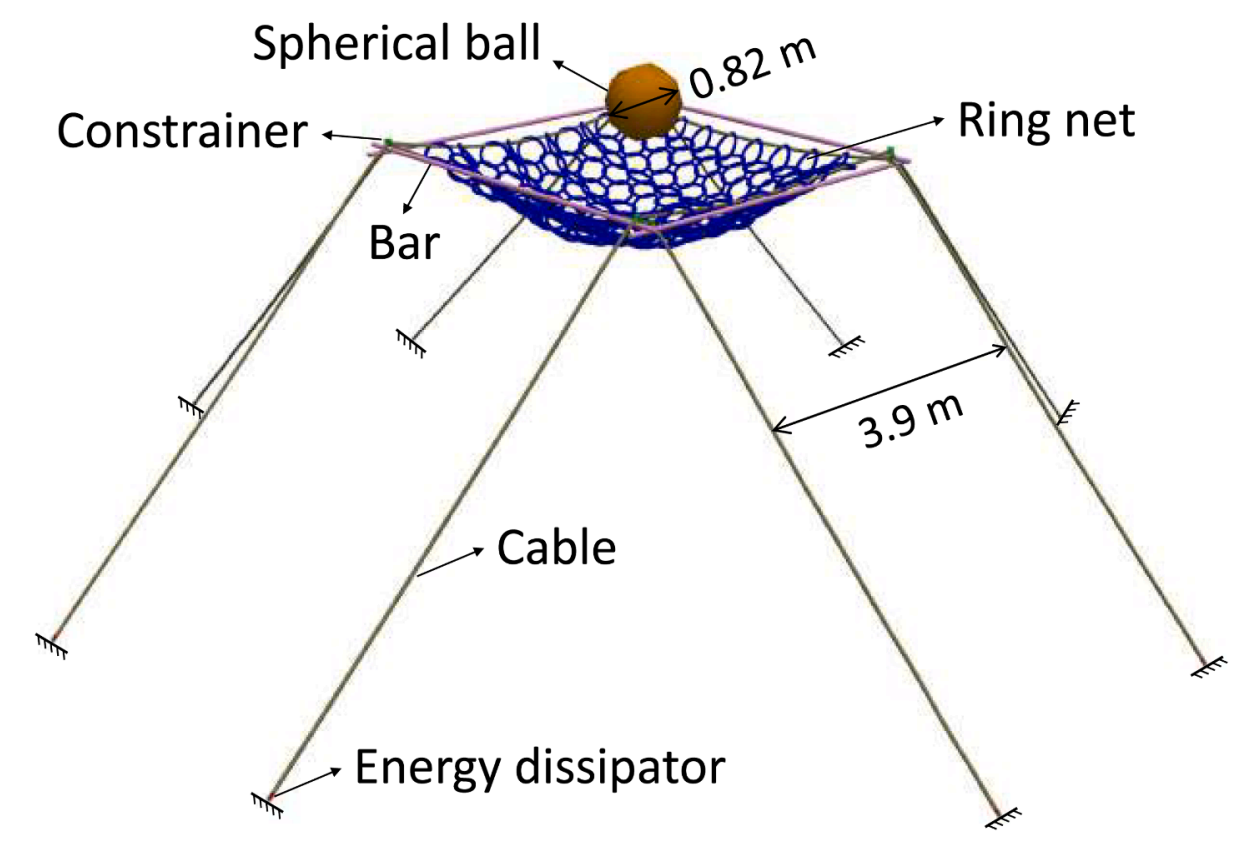

(b)

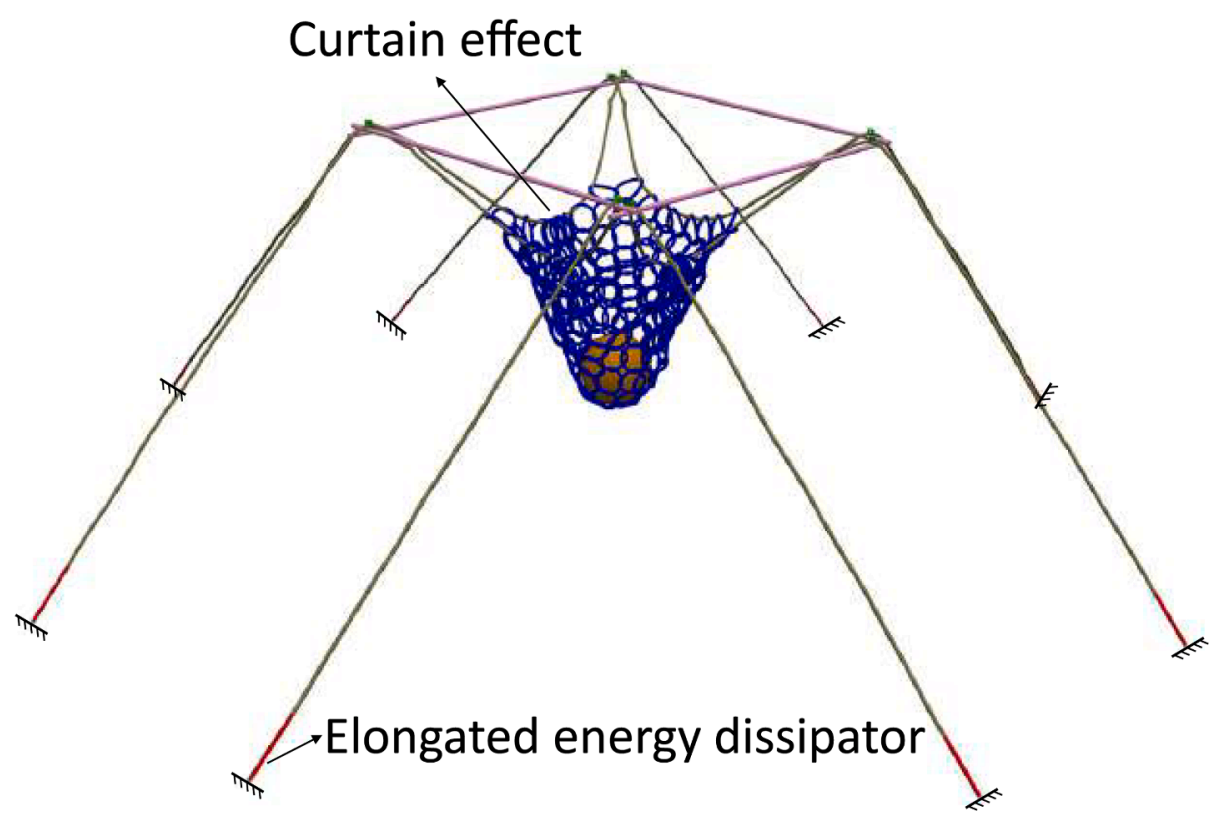

Fig. 6. (a) DEM setup for modeling rockfall impact on a flexible ring net barrier. (b) Curtain effect observed during the rockfall impact.

\section{Debris flow impacts on flexible ring net barriers}

\subsection{Model setup}

Fig. 8 shows the model setup used in this study to simulate the impacting of debris flow on a flexible ring net barrier. The simulation domain is bounded by a cuboid with 6 faces (i.e., $x_{1}, x_{2}, y_{1}, y_{2}, z_{1}, z_{2}$ ). The boundary planes $x_{1}, y_{2}, z_{1}, z_{2}$ are set as walls to form a chute. The planes $x_{2}$ and $y_{1}$ are set to be atmosphere and outlet, respectively. The numerical settings for different boundary conditions in the CFD and the
DEM follow our previous study (Li and Zhao, 2018). A debris mixture of water and a bi-dispersed particle system is initially placed on the top of the channel, leaving the rest of the simulation domain filled with air. Under gravity, the debris mixture is released to flow down and impact on the flexible ring net barrier situated at a distance of $L$ from the top end of the mixture. The flexible ring net barrier consists of rings, cables and energy dissipators. The diameter of the rings is $0.07 \mathrm{~m}$, which is designed to block the large debris particles while allowing the small debris particles to pass through. Each ring is connected with four surrounding rings according to a typical ring net barrier as demonstarted in 
Table 3

Adopted parameters in the rockfall simulation.

\begin{tabular}{|c|c|c|c|c|c|}
\hline & & Ball & Ring & Cable & $\begin{array}{l}\text { Energy } \\
\text { dissipator }\end{array}$ \\
\hline \multirow[t]{4}{*}{ Particle } & Density $\left(\mathrm{kg} / \mathrm{m}^{3}\right)$ & 2858 & 7800 & 7800 & 7800 \\
\hline & $\begin{array}{l}\text { Young's modulus } \\
\text { (GPa) }\end{array}$ & 30 & 100 & 100 & 100 \\
\hline & Poisson's ratio & 0.15 & 0.3 & 0.3 & 0.3 \\
\hline & $\begin{array}{l}\text { Friction } \\
\text { coefficient }\end{array}$ & 0.1 & 0.1 & 0.1 & 0.1 \\
\hline \multirow[t]{2}{*}{ Bond } & Stiffness (N/m) & N/A & $\begin{array}{l}3.0 \times \\
10^{11}\end{array}$ & $\begin{array}{l}8.0 \times \\
10^{11}\end{array}$ & $\begin{array}{l}3.5 \times 10^{8} ; 3.5 \times \\
10^{7}\end{array}$ \\
\hline & $\begin{array}{l}\text { Activation force } \\
(\mathrm{kN})\end{array}$ & N/A & N/A & N/A & 12 \\
\hline
\end{tabular}

Fig. 1. The two ends of all the cables and the middle of the lateral cables are fixed to duplicate the anchored boundary conditions in reality (Wendeler et al., 2018b). In addition, the bottom cable is completely fixed to the channel bed to avoid excessive debris particles escaped from the barrier bottom (Brighenti et al., 2013; Albaba et al., 2017). Detailed parameters adopted in the simulations are summarized in Table 4. Please note that the time step of DEM is constrained by the Rayleigh time (Smuts et al., 2012), whereas the time step of CFD refers to the typical coupling interval between CFD and DEM (Goniva et al., 2010; Derakhshani et al., 2016). In this study, the CFD and DEM computations exchange information every 10 time steps of the DEM to guarantee accuracy and efficiency of the simulations. The CFD cell size is determined based on the Sauter-mean diameter of the particles (Volk et al., 2018).

(a)

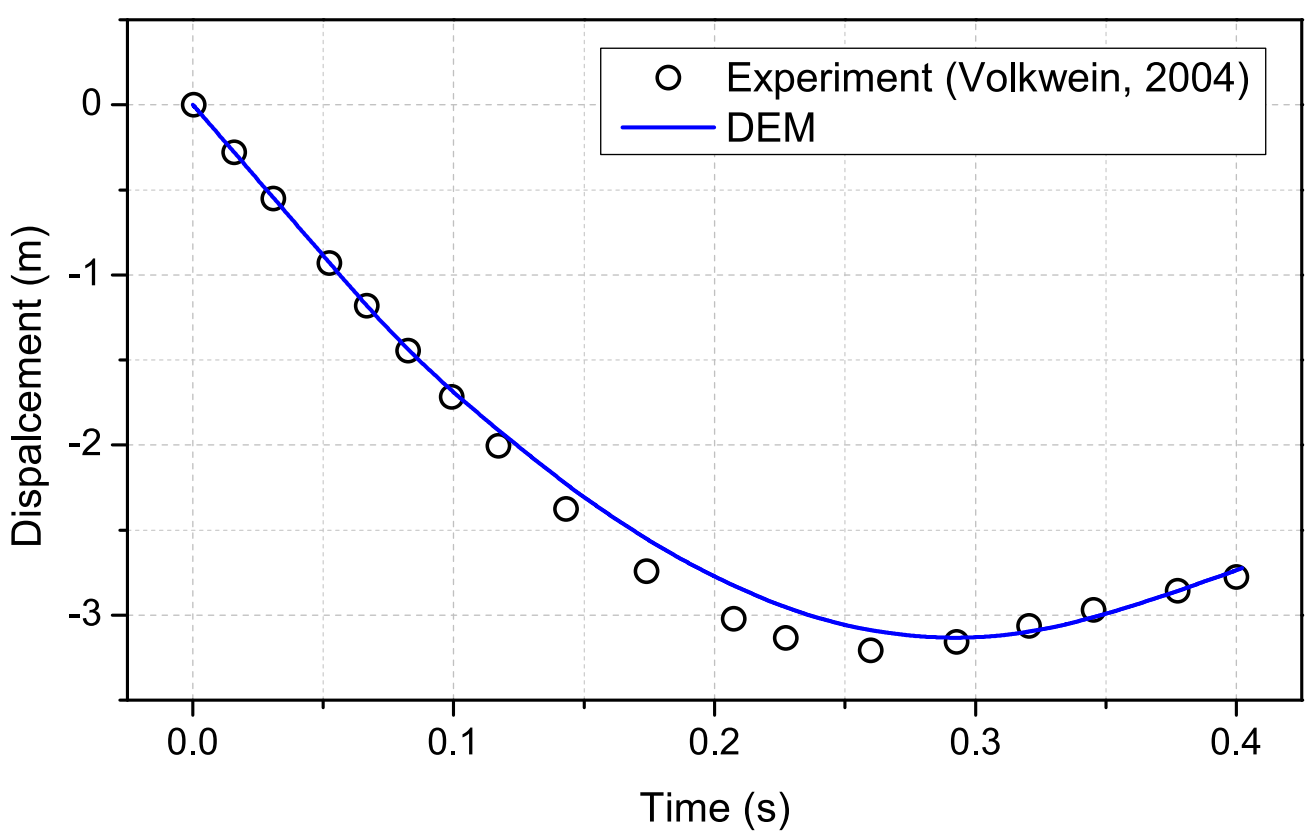

(b)

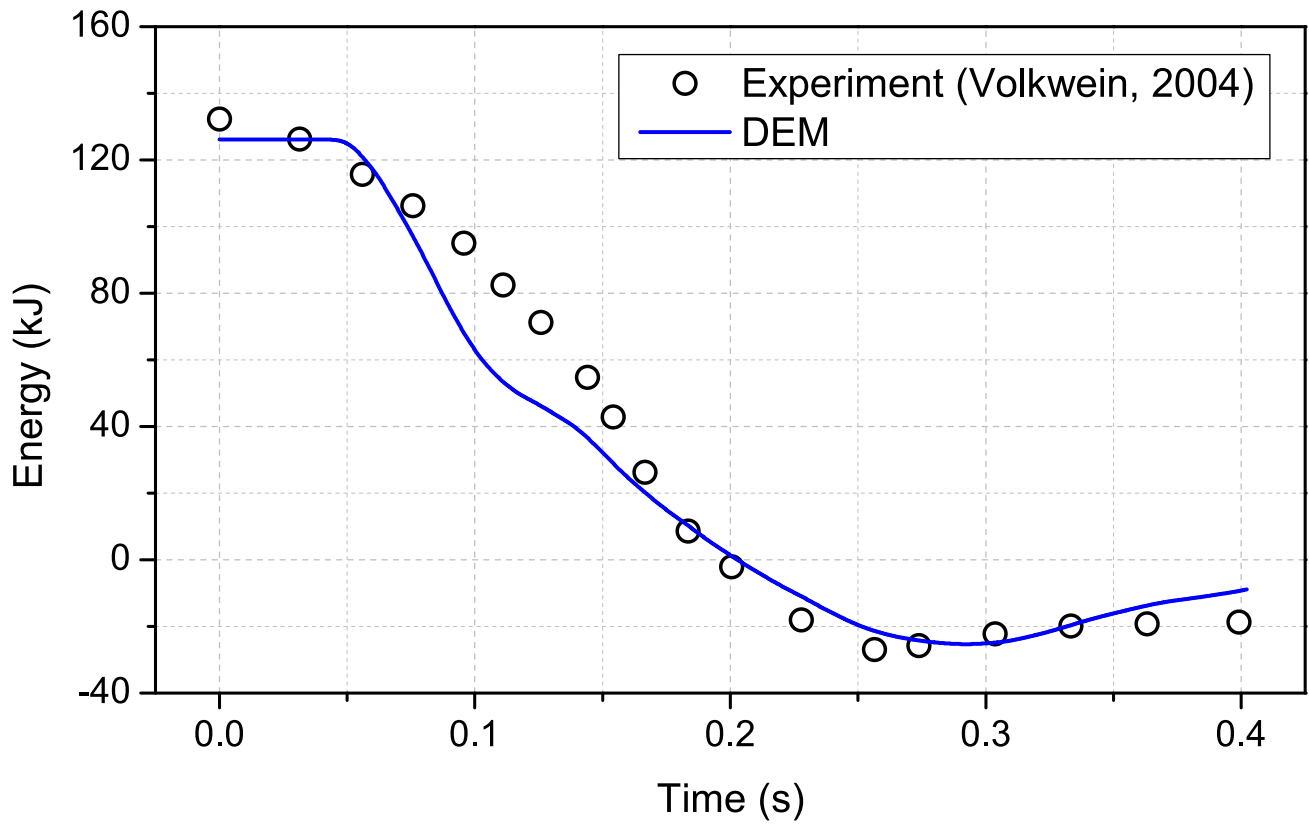

Fig. 7. Evolution of (a) displacement and (b) energy of the spherical ball in the simulation of rockfall impact on a flexible ring net barrier. 


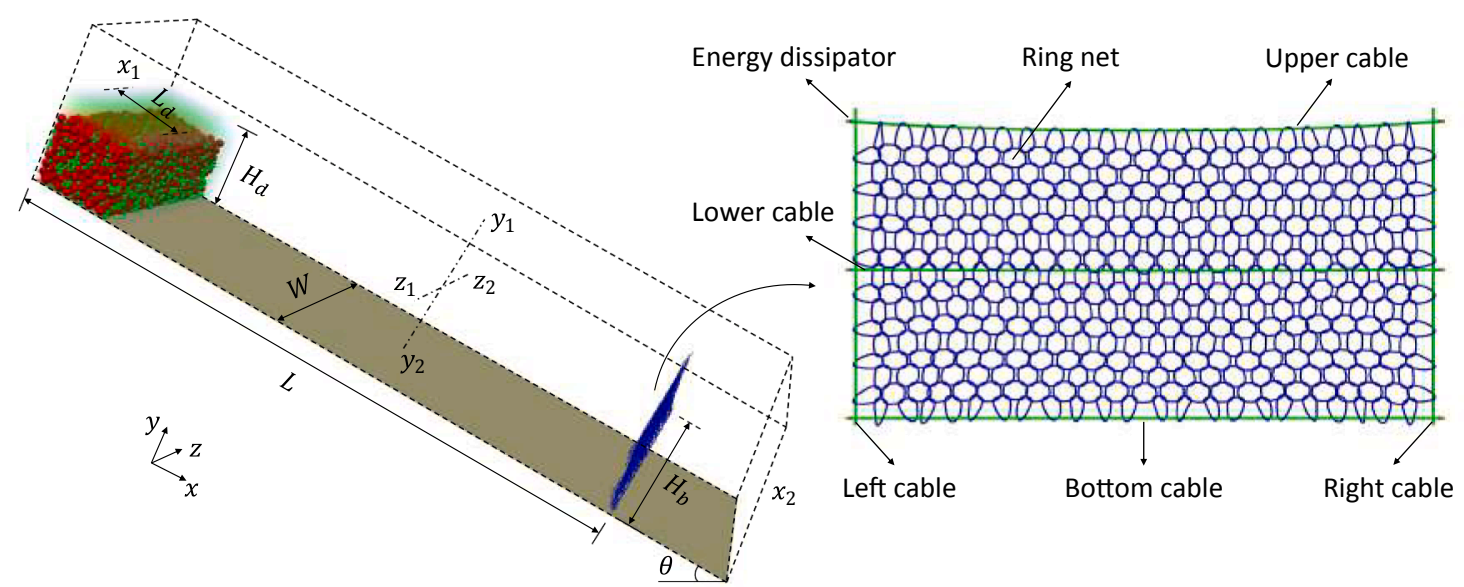

Fig. 8. Setup of CFD-DEM simulation for modeling debris flow impacts on a flexible ring net barrier. The surface normals of the $x_{1}, x_{2}$ planes, $y_{1}, y_{2}$ planes, and $z_{1}, z_{2}$ planes are along the $x, y, z$ directions, respectively.

Table 4

Model parameters adopted in CFD-DEM simulations of debris flow impacts on a flexible ring net barrier.

\begin{tabular}{|c|c|c|}
\hline \multirow[t]{8}{*}{ Debris particle } & Diameter* & $0.04 \mathrm{~m}, 0.08 \mathrm{~m}$ \\
\hline & Density & $2500 \mathrm{~kg} / \mathrm{m}^{3}$ \\
\hline & $\begin{array}{l}\text { Young's modulus (particle-particle } \\
\text { contact) }\end{array}$ & $70 \mathrm{GPa}$ \\
\hline & Young's modulus (particle-wall contact) & $700 \mathrm{GPa}$ \\
\hline & Poisson's ratio & 0.3 \\
\hline & Restitution coefficient & 0.7 \\
\hline & Interparticle friction coefficient & 0.7 \\
\hline & Particle-wall friction coefficient & 0.7 \\
\hline \multirow[t]{2}{*}{ Water } & Density & $1000 \mathrm{~kg} / \mathrm{m}^{3}$ \\
\hline & Viscosity & $0.001 \mathrm{~Pa} \cdot \mathrm{s}$ \\
\hline \multirow[t]{3}{*}{ Air } & Density & $1 \mathrm{~kg} / \mathrm{m}^{3}$ \\
\hline & Viscosity & $1.48 \times 10^{-5}$ \\
\hline & & $\mathrm{Pa} \cdot \mathrm{s}$ \\
\hline \multirow[t]{6}{*}{ Barrier particles } & Particle diameter & $0.0061 \mathrm{~m}$ \\
\hline & Particle density & $7800 \mathrm{~kg} / \mathrm{m}^{3}$ \\
\hline & $\begin{array}{l}\text { Young's modulus (particle-barrier } \\
\text { contact) }\end{array}$ & $10 \mathrm{GPa}$ \\
\hline & Poisson's ratio & 0.3 \\
\hline & Restitution coefficient & 0.1 \\
\hline & Interparticle friction coefficient & 0.1 \\
\hline \multirow[t]{5}{*}{ Barrier bonds } & Stiffness of rings ${ }^{* *}$ & $3 \times 10^{11} \mathrm{~N} / \mathrm{m}$ \\
\hline & Stiffness of cables ${ }^{* *}$ & $8 \times 10^{11} \mathrm{~N} / \mathrm{m}$ \\
\hline & $\begin{array}{l}\text { Stiffness of energy dissipators at Stage } 1 \\
\bar{k}_{1}^{n * *}\end{array}$ & $8 \times 10^{11} \mathrm{~N} / \mathrm{m}$ \\
\hline & Activation force of energy dissipators $\bar{F}_{1}^{n}$ & $2 \mathrm{kN}$ \\
\hline & $\begin{array}{l}\text { Stiffness of energy dissipators at Stage } 2 \\
\bar{k}_{2}^{n} \ldots *\end{array}$ & $8 \times 10^{10} \mathrm{~N} / \mathrm{m}$ \\
\hline \multirow[t]{5}{*}{ Geometric size } & $\begin{array}{l}\text { Width of debris mixture and barrier } W \\
\text { Length of debris mixture } L_{d}\end{array}$ & $\begin{array}{l}1.8 \mathrm{~m} \\
0.75 \mathrm{~m}\end{array}$ \\
\hline & Height of debris mixture $H_{d}$ & $0.75 \mathrm{~m}$ \\
\hline & Barrier height $H_{b}$ & $0.96 \mathrm{~m}$ \\
\hline & Release distance $L$ & $1.85 \sim 8.6 \mathrm{~m}$ \\
\hline & Slope angle $\theta$ & $35^{\circ}$ \\
\hline \multirow{4}{*}{$\begin{array}{l}\text { Simulation } \\
\text { control }\end{array}$} & Time step (CFD) & $5 \times 10^{-6} \mathrm{~s}$ \\
\hline & Time step (DEM) & $5 \times 10^{-7} \mathrm{~s}$ \\
\hline & Simulated real time & $4.0 \mathrm{~s}$ \\
\hline & Cell size (CFD) & $0.15 \mathrm{~m}$ \\
\hline
\end{tabular}

* A bi-disperse particle system is considered here, where the numbers of particles with $0.04 \mathrm{~m}$ diameter and $0.08 \mathrm{~m}$ diameter are 3260 and 1481, respectively. The bulk density of the initial particle packing is $1607 \mathrm{~kg} / \mathrm{m}^{3}$.

The normal stiffness is assumed to be identical to the shear stiffness for all the barrier components.
By placing the debris mixture at different locations (i.e., changing $L$ in Fig. 8), inflowing debris mass with different Froude numbers can be examined. Froude number $\mathrm{Fr}$ is defined as the ratio of inertia field to external field (gravitational field in this study), which has been closely related to the impact behavior of debris flow (Hübl et al., 2009; Vagnon, 2020). As expressed by Eq. 2, Fr reflects two key flow characteristics, flow velocity $v$ and flow height $h$.

$F r=\frac{v}{\sqrt{g h \cos \theta}}$

The Fr of real-scale debris flows is usually smaller than 2, while smallscale experiments and numerical modeling normally give a larger $\mathrm{Fr}$ (Hübl et al., 2009; Scheidl et al., 2013). In this study, the range of Fr is from 0.41 to 7.09. Following Li et al. (2020), the flow velocity and flow height are extracted from a section of debris mass before the debris mixture impacts on the flexible barrier, by which the velocity of the front (Hu et al., 2011; Scheidl et al., 2013) and the height of the main body can be reflected (Li et al., 2020). The flow height is determined by the free surface of the liquid phase, since the debris particles are largely submerged in the debris liquid before the impact.

\subsection{Retained debris mass}

A natural debris flow commonly consists of water, fine debris particles (e.g., clay and silt), and coarse debris particles (e.g., gravels, boulders). Using a flexible barrier, the coarse particles can be effectively intercepted, while the debris liquid and fine debris particles are allowed to pass through the barrier. In this study, all water in the debris mixture will pass through the flexible barrier after the debris impact, while a portion of the debris particles (including both coarse and fine particles) are retained by the flexible barrier as demonstrated in Fig. 9.

The ratio of the mass of the retained debris particles $M_{p}$ to the overall mass of the debris particles before the impact $M_{p 0}$ decreases from 0.98 to 0.90 with an increasing Froude number Fr as illustrated in Fig. 10. This negative relation reveals that a debris flow with a large $F r$ has less debris mass blocked by the flexible barrier. Indeed, a large $\mathrm{Fr}$ denotes a high flow velocity, leading to more intense impact on the flexible barrier and more magnification of the barrier openings. Consequently, it is easier for debris particles to pass through the flexible barrier. In addition, as the debris mass is relatively difficult to be intercepted and accumulated behind the barrier, the reduction in barrier permeability is slower, which further causes more debris mass escaped from the flexible barrier. In the CFD-DEM simulations, there are both large and small particles that escape the interception by either passing through or overtopping the flexible barrier. The blocked large particles are $96.5 \% \sim 99.9 \%$ of 
(a)

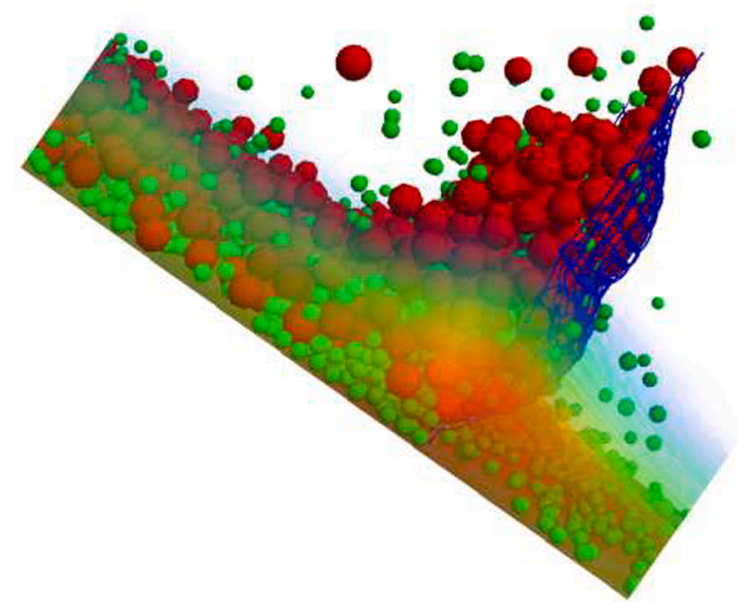

(b)

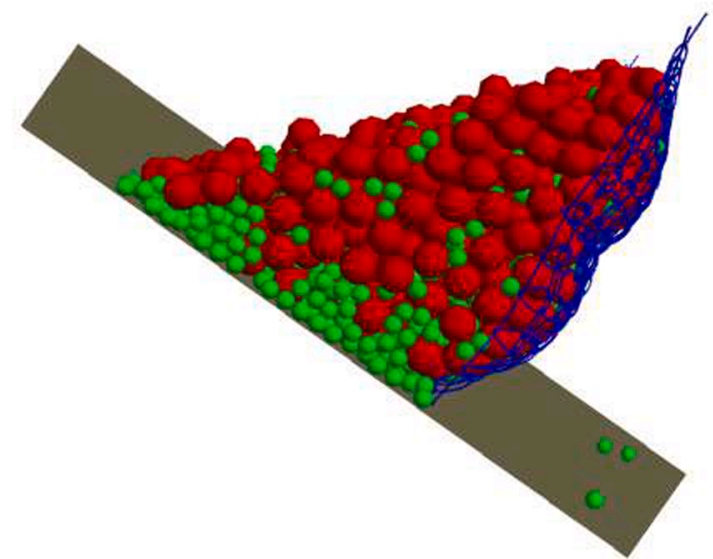

Fig. 9. Retained debris mass in a representative case with $F r=3.111$ at (a) $t=1.5 \mathrm{~s}$ and (b) $t=3.8 \mathrm{~s}$.

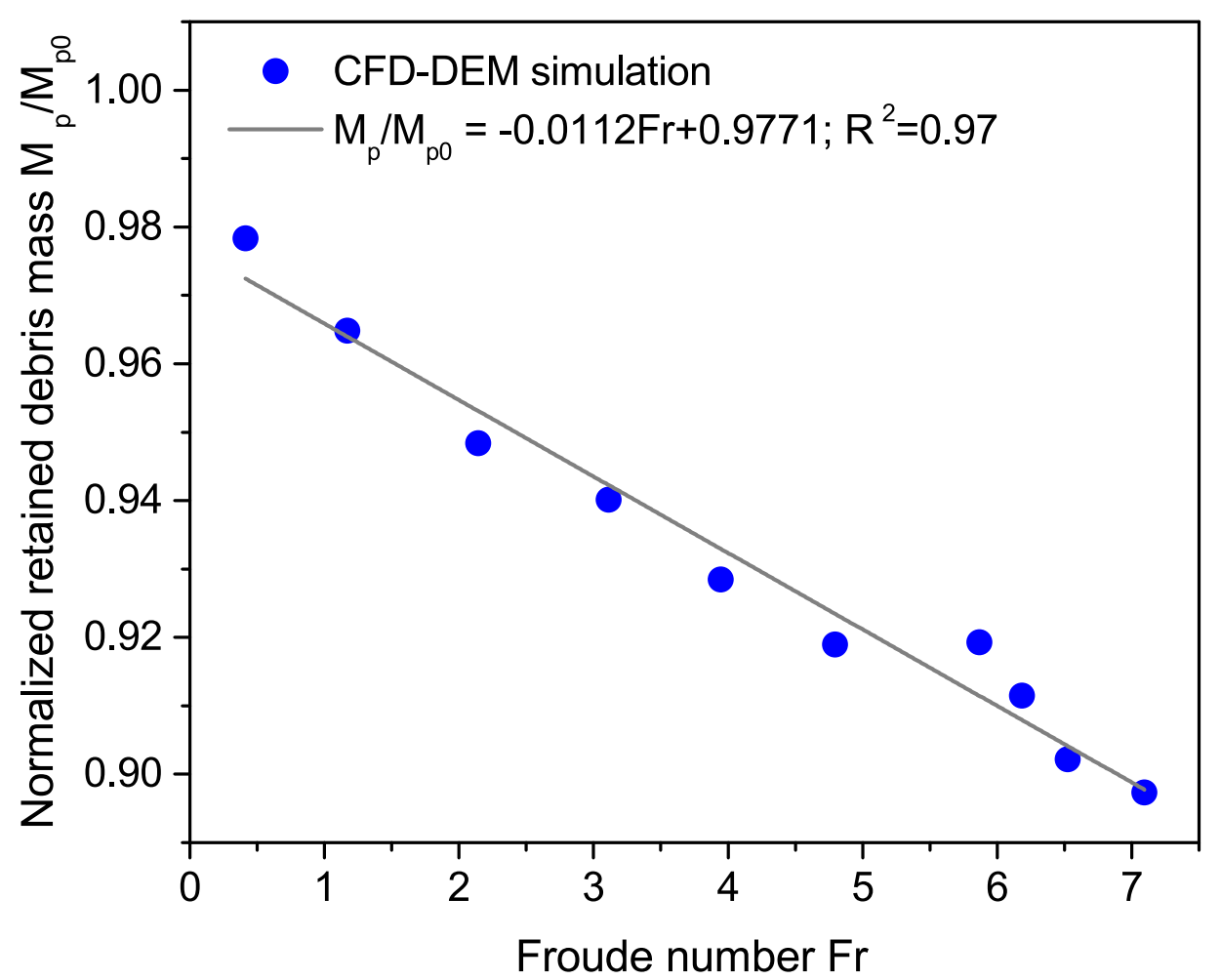

Fig. 10. Normalized retained debris mass for debris flows with different Froude numbers. $M_{p 0}$ is the mass of the debris particles before the impact. $M_{p}$ is the mass of the debris particles retained by the flexible barrier after the impact.

their initial mass, whilst the retained small particles are $64.9 \% \sim 90.2 \%$ of the initial small particles. Indeed, the large particles are much more effectively arrested in comparison with the small particles. The escaped small particles mainly pass through the barrier from the lower part as shown in Fig. 9a, due to the reverse grading of the granular system. The retained small particles are primarily at the bottom of the granular packing as well (see Fig. 9b).

\subsection{Force sustained in the flexible barrier}

The design of rigid barriers can be well based on the impact force (Kwan, 2012). In comparison, the force analysis of a flexible barrier is more complicated, due mainly to the barrier deformability, the force rearrangement/transfer among barrier components and the distinct strengths of different barrier components. Fig. 11 shows the deformed rings and the sliding of the rings during the impact process, where the light and dark pictures denote the early and later stages of the impact, respectively. As highlighted by the black ellipse in Fig. 11a, a ring can be highly deformed during the impact. In addition, rings attached to a cable can slide (see Fig. 11b), which rearranges the barrier shape and redistributes the load in the barrier. It is thus essential to examine the force distribution within the barrier and the force sustained in key barrier components.

Fig. 12 demonstrates two typical patterns of the force distribution in a flexible ring net barrier subjected to the dynamic impact of a debris mixture, where the local force is the force in each parallel bond. In both 
(a)

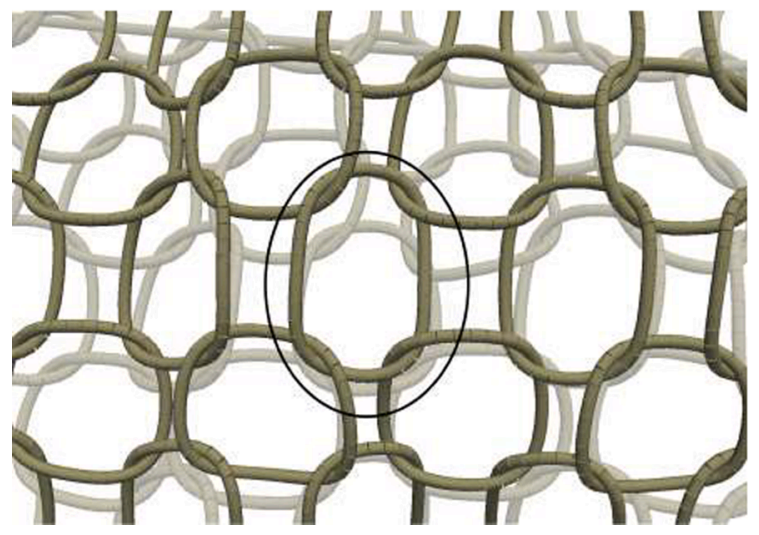

(b)

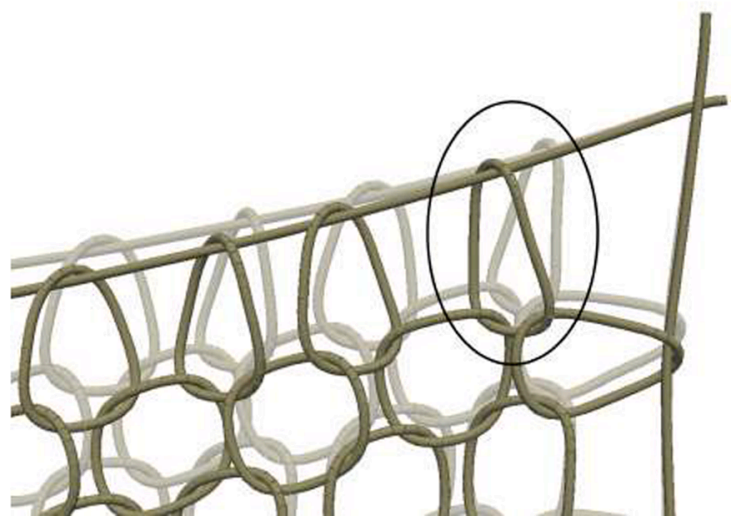

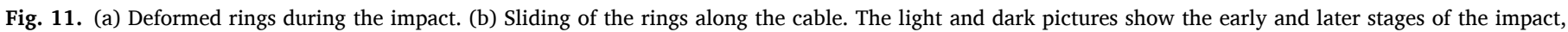
respectively. Black ellipses highlight a deformed ring in (a) and a sliding ring in (b).

(a)

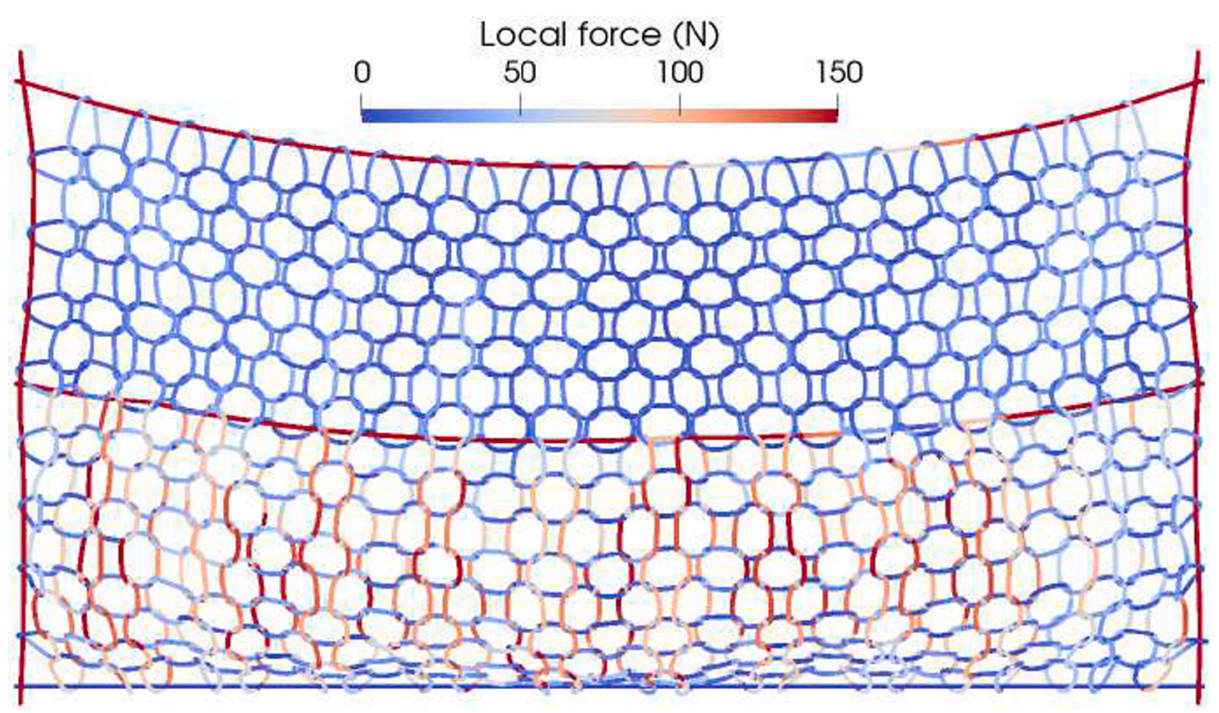

(b)

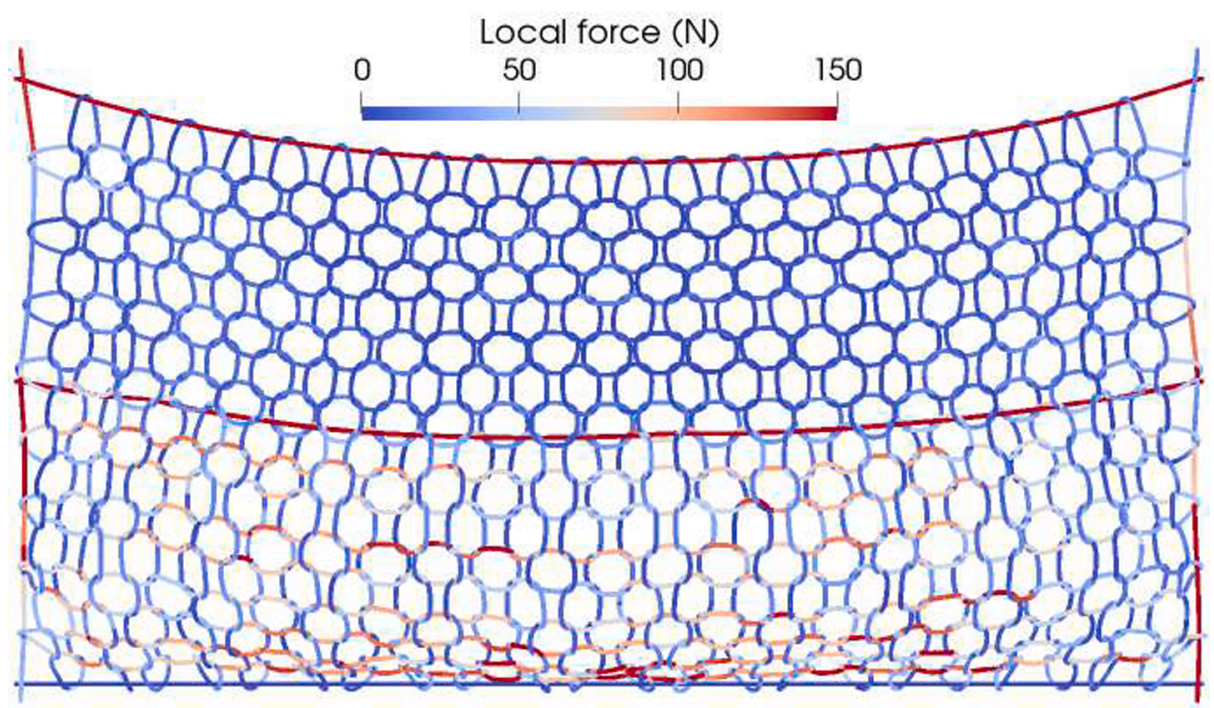

Fig. 12. Force distribution within a flexible ring net barrier in a representative case with $\mathrm{Fr}=3.111$ at $(\mathrm{a}) \mathrm{t}=1.205 \mathrm{~s}$ and $(\mathrm{b}) \mathrm{t}=1.210 \mathrm{~s}$. 
patterns, the maximum local force is sustained by the cables, indicating the effective load transfer of the impact from the rings to the cables. This may partially prove that ring nets may be more efficient than twisted wire nets in resisting and redistributing the impacting forces for practical debris flow mitigations. Moreover, larger forces are observed in the rings at the lower part of the barrier, since the incoming debris mass keeps impacting the lower part and the gravity of the deposited debris mass is mainly sustained by the lower part as well. The pattern in Fig. 12a shows the vertical chains of strong forces in the rings, whose occurrence reflects the vertical stretching of the ring net. In addition, horizontal chains of strong forces are also observed as shown in Fig. 12b, where the ring net is subjected to lateral stretching. Based on all simulated cases, it is observed that the pattern with the vertical chains dominates the entire impact process, including the initial dynamic impact and the final stationary state. Indeed, the impacting particles predominantly climb up if not deposit or pass through the barrier, imposing forces to the barrier along the vertical direction. Furthermore, the deposited particles also contribute to the vertical stretching of the ring net due to their gravity. Compared with the barrier net composed of diamond meshes in Albaba et al. (2017) and the barrier net consisting of hexagonal meshes in Li and Zhao (2018), no obvious chains of large forces are observed along the diagonal directions of the barrier ring net in this study, which might be due to the effects of mesh shape and mesh connection method. As sliding and collision are allowed among the interlocking rings, the force within a ring is easier to be transfered to the surrounding rings, whilst its effect on relatively distant rings become feeble. The large force at the lower part of the ring net is not effectively transferred to the upper corners of the ring net, leading to the absence of the chains along the diagonal directions.

Cables play a crucial role in supporting the barrier net and transferring impact loads to the boundaries. In this study, the maximum local force in all the simulated cases is concentrated on the cables, consistent with the findings in Albaba et al. (2017) and Li and Zhao (2018). The local force distribution within the cables (as shown in Fig. 12) does not follow a clear pattern during the impact, which might be due to the chaotic collisions from the debris particles as well as the sliding and collisions from the surrounding rings. Fig. 13 illustrates the normalized total force sustained in the four cables of the barrier (see Fig. 8) in a representative case. The total force in a cable $F^{\text {cable }}$ is defined as the sum of the forces of all the parallel bonds forming the cable. At the initial stage $(t=0 \sim 0.75 \mathrm{~s})$, minor fluctuations in forces are observed before the debris mixture impacts on the barrier, due to the gravity and the elasticity of the barrier itself. These initial fluctuations are more notable in the horizontal cables (lower and upper) which play a major role in preventing the falling of the ring net under gravity. At $t=0.75 \mathrm{~s}$, the debris liquid at the front of the flow impacts on the barrier, inducing small local peaks at around $t=0.83 \mathrm{~s}$ in the lower, left and right cables and $t=0.94 \mathrm{~s}$ in the upper cable. The lagging of the force increase in the upper cable reflects the time needed for the load transfer from the lower part to the upper part of the barrier. The higher local peak force in the upper cable might be due to the gravity of more rings supported. The debris particles reach the barrier at $\mathrm{t}=0.92 \mathrm{~s}$, after which significant increases of forces occur in the lower, left and right cables. The force growth in the upper cable happens at around $t=1.04 \mathrm{~s}$. During the dynamic impact, the debris mass dominantly moves along stream-wise and bed-normal directions, with an insignificant lateral motion. Consequently, the forces in the lower and upper cables are much higher than that in the left and right cables. The highest force occurs in the lower cable, which agrees with the field measurements (Bugnion and Wendeler, 2010) when no severe overtopping happens. Unlike other three cables where only one major bulge is observed during the dynamic impact from $\mathrm{t}=0.92 \mathrm{~s}$, the upper cable demonstrate two major bulges after $\mathrm{t}=1.04 \mathrm{~s}$. The first bulge $(\mathrm{t}=1.04 \sim 1.61 \mathrm{~s})$ is resulted from the load transferred from the connected rings, whilst the second bulge $(\mathrm{t}=$ $1.61 \sim 2.36 \mathrm{~s}$ ) is chiefly due to the direct contacts between the upper cable and the debris particles as the debris mass reaches the top of the barrier. After $t=2.36 \mathrm{~s}$, the forces in the cables tend to be stable as the debris mass become stationary.

Further cases of debris mixtures with different Froude numbers are explored to examine the peak total force sustained by the cables $F_{\text {peak }}^{\text {cable }}$.

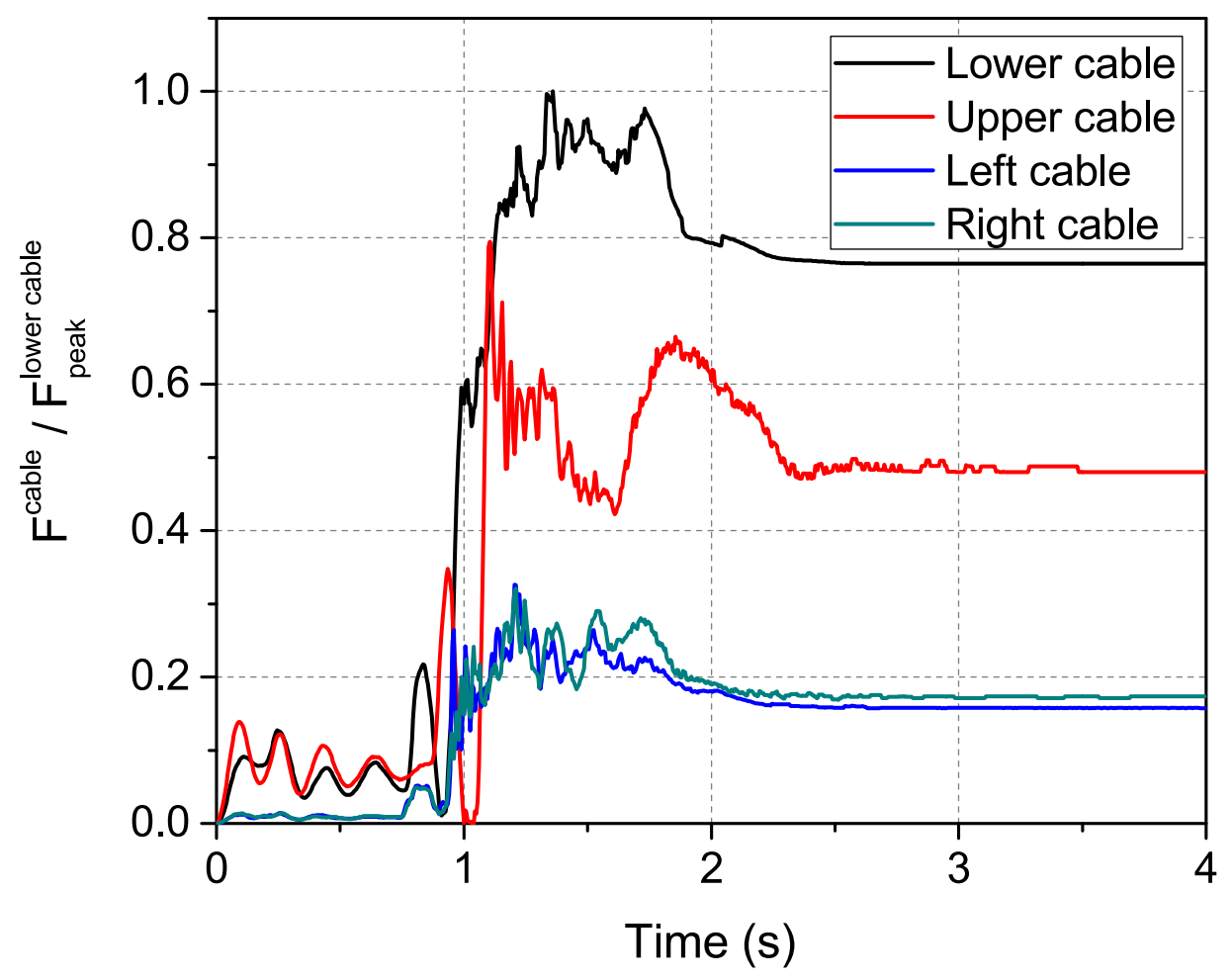

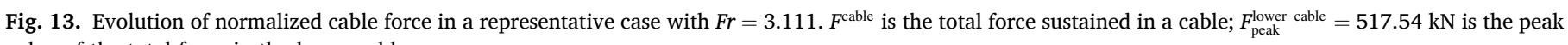
value of the total force in the lower cable. 


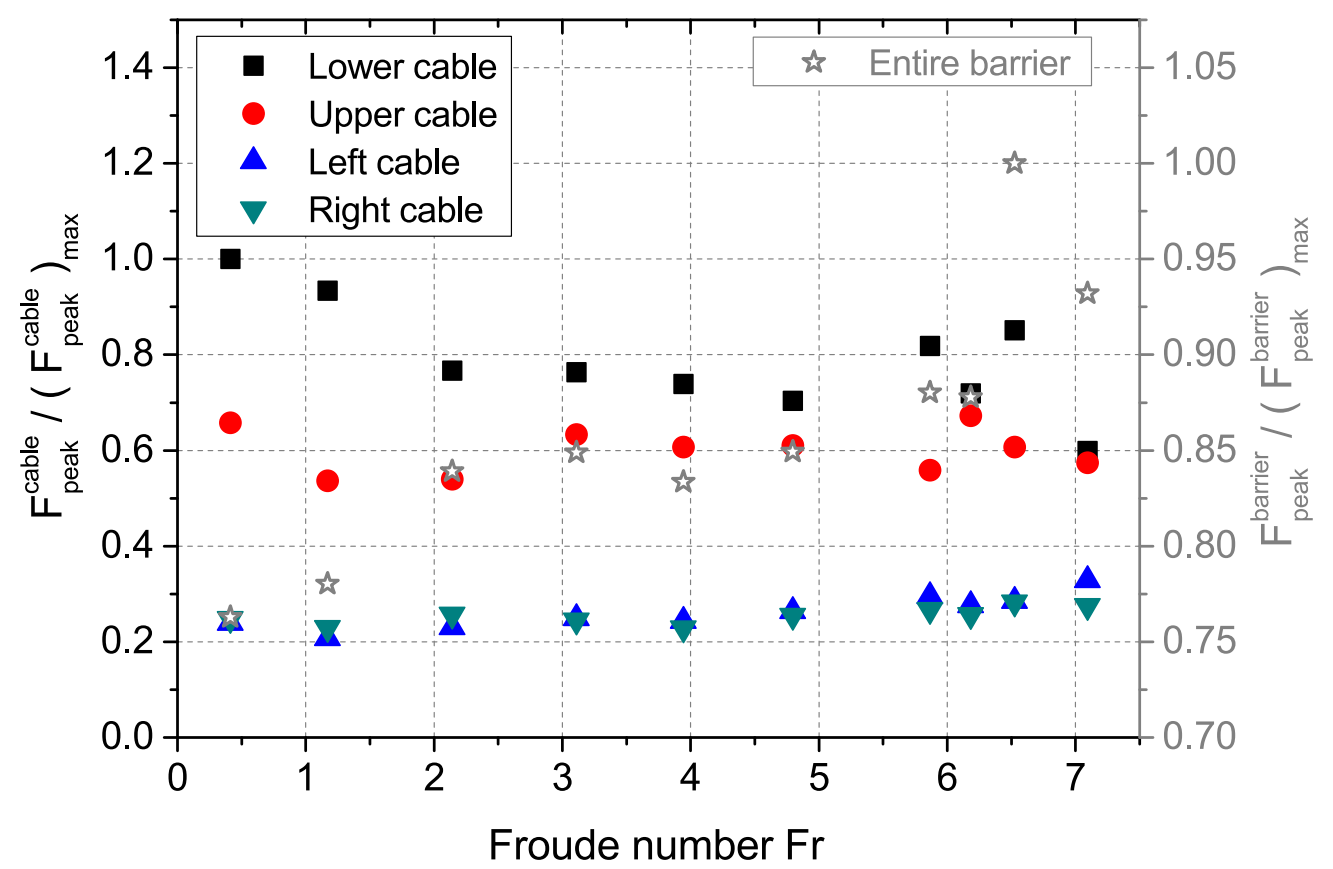

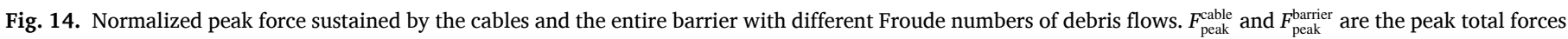

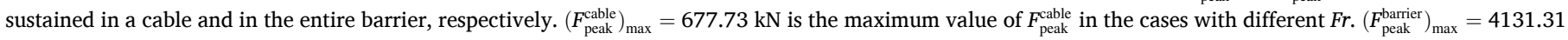
$\mathrm{kN}$ is the maximum value of $F_{\text {peak }}^{\text {barrier }}$ in the cases with different $F r$.

The results are summarized in Fig. 14, along with the peak total force sustained in the entire barrier $F_{\text {peak }}^{\text {barrier }}$. The peak total force in a cable and an entire barrier are defined as the peak value of the total force sustained by the cable and the barrier, respectively. $F_{\text {peak }}^{\text {cable }}$ and $F_{\text {peak }}^{\text {barrier }}$ are normalized in Fig. 14 by their maximum values obtained from all the cases with different Froude numbers. As demonstrated in Fig. 14, the lower cable tends to possess a smaller peak total force with the increase of $F r$, whilst the other three cables do not show significant variations. As the lower cable endures the most critical force compared with the other three cables, it is more sensitive to the change of the flow behaviors (velocity and height). Nevertheless, it is interesting that $F r$ tends to be negatively correlated with the peak total force in the lower cable, in contrast to a positive correlation with the peak total force in the entire barrier. The increase of $\mathrm{Fr}$ indicates the growth of debris velocity before the impact, which consequently leads to more intense impacts and thus a larger peak total force in the barrier. Nevertheless, the larger force in the entire barrier does not necessarily result in a larger force in the cable, since the debris mass is mainly in contact with the rings. With a higher $\mathrm{Fr}$ and more intense impacts on the interlocking rings, the interactions among the rings (e.g., frictional sliding, collision) might be more violent to dissipate more impact energy, leading to smaller forces transferred to the cables. In addition, the peak total force is attained after notable debris mass has impacted on the barrier, before which the reflected debris flow helps dissipate the energy of the incoming flow. The higher Fr may render higher velocity of the reflected flow, which may induce more energy dissipation of the incoming flow and results in the smaller force in the lower cable. The distinct trends for the cables and the entire barrier in Fig. 14 highlight the great importance to analyze the force distribution within a barrier and the force in key barrier components for the barrier design, in addition to the overall force in the entire barrier.

\subsection{Energy dissipation of debris flow and energy absorption by flexible barrier}

In the absence of overtopping, a solid rigid barrier retains all the incoming debris mass, and completely dissipates the debris energy without notable barrier deformation. In contrast, a flexible barrier allows the passage of debris fluid and fine debris particles, reducing the energy that has to be dissipated by the barrier. Moreover, the deformability of a flexible barrier helps to enhance the energy dissipation process and facilitates the absorption of debris energy by barrier deformation. To examine the performance of a flexible barrier, it is essential to study the dissipation of debris energy and the absorbed energy by the barrier. Using a flexible barrier to mitigate a debris flow, the energy of incoming debris flow $E_{i n}$ can be mainly dissipated and transmitted by 1) internal friction and collision, 2) interaction with boundaries (e.g., river bank and bed), 3) impaction on the barrier. According to energy conservation, the dissipated and transferred debris energy $E_{d}$ can be obtained as follows

$E_{d}=E_{\text {in }}-E_{\text {out }}$

where $E_{\text {out }}$ is the energy of outflow. The absorbed debris energy by the flexible barrier $E_{b}$ is part of the energy transmitted from the debris flow to the barrier, leaving the rest dissipated during the transmission.

The ratio $E_{b} / E_{\text {in }}$ has been widely used to reflect the efficiency of a flexible barrier (Ng et al., 2016; Huo et al., 2018; Kwan et al., 2018; Song et al., 2019). To better illuminate the impact mechanisms, two terms in consideration of the outflow energy are studied, namely, the energy dissipation ratio $\eta$ and the energy absorption ratio $\zeta$ defined as follows

$\eta=\frac{E_{d}}{E_{\text {in }}}$

$\zeta=\frac{E_{b}}{E_{d}}$

The energy dissipation ratio $\eta$ quantifies the efficiency of debris energy dissipation with the installation of a flexible barrier. The higher the $\eta$, the more efficient the flexible barrier is. The energy absorption ratio $\zeta$ assesses how much energy the barrier needs to absorb given the dissipated energy. The higher the $\zeta$, the less efficient the flexible barrier is. Although $\zeta$ is defined in terms of energy, it also reflects barrier deformation since a flexible barrier stores energy mainly by deformation. Moreover, the sustained force by the barrier can be further estimated based on the barrier deformation. Given a fixed amount of energy 
dissipation, a higher $\zeta$ indicates more stored energy, large barrier deformation and high barrier force, and thus a less effective flexible barrier. Note the efficiency of the flexible barrier here is only evaluated in terms of energy, which should surely be considered in conjunction with other perspectives including installation cost and maintenance cost in practical design. If the barrier is to be installed in hard-to-access regions where the maintenance cost is high, a barrier with a low $\zeta$ is suggested because the probability of maintenance is low. Nevertheless, the low $\zeta$ may meanwhile indicate a stronger barrier and a higher installation and construction cost.

In this study, the energy of incoming debris flow $E_{\text {in }}$ is calculated by summing up the kinetic and potential energy of the debris mixture when the distance between the flow front and the barrier decreases to the size of one CFD cell $(0.15 \mathrm{~m})$. The datum line to calculate the potential energy is located at the base of the flexible barrier. The energy of outflow $E_{\text {out }}$ is obtained by accumulating the kinetic and potential energy of the debris mass at a cross section located $0.325 \mathrm{~m}$ downstream of the barrier. The $0.325 \mathrm{~m}$ is determined based on the maximum barrier elongation from the simulations $(0.263 \mathrm{~m}$ along the $x$ direction in Fig. 8$)$ and the CFD cell center ( $0.325 \mathrm{~m}$ downstream of the barrier). The energy absorbed by the barrier $E_{b}$ is calculated as the changed barrier energy before and after debris flow impact, including potential energy and elastic energy of the barrier. The change of its kinetic energy is close to zero, since the barrier is largely stable before the debris mass reaches the barrier and after the retained debris becomes stationary. For all the simulated cases in this study, the decreased barrier potential energy due to debris flow impact is trivial, which is $0.3 \% \sim 0.5 \%$ of the increased elastic energy of the barrier. The elastic energy of the barrier is calculated by the force and the elongation of all the parallel bonds forming the barrier. Table 5 summarizes the energy-related data obtained from the simulations.

Fig. 15 shows evolution of the energy dissipation ratio $\eta$ with Froude number Fr. With the increase of $F r, \eta$ increases before tending to be stable. The initial increase indicates more debris energy dissipated by the flexible barrier. Indeed, a large Fr hints a large flow velocity, which, intuitively, may lead to more energy dissipation of a debris flow due to three aspects. First, collisions between the debris mass and boundary walls become intense. Secondly, the debris front may need to roll back after it impacts on the flexible barrier. The higher the flow velocity prior to the impact, the higher the velocity of the reflected flow. This reflected flow with a high velocity can help to dissipate more energy of the encountered incoming flow. Thirdly, a higher flow velocity leads to a higher impact force on the barrier, which causes larger barrier deformation and more absorbed debris energy by the barrier. The growth of $\eta$ with Fr in Fig. 15 vanishes when Fr becomes high ( $\approx 5.866$ ), which might be due to the small flow height with a high Fr. Considering a fixed flow velocity, the smaller the flow height is, the smaller the impact area on the barrier becomes, which leads to less energy to be absorbed by the barrier and less reflected debris mass to dissipate the energy of subsequently incoming flow. Thus, a smaller flow height results in less energy dissipation of the debris flow. The stable stage in Fig. 15 might be due to the combined effect of a higher flow velocity and a small flow height

Table 5

Energy-related data from the simulations.

\begin{tabular}{lllllll}
\hline Fr & $E_{\text {in }}(\mathrm{kJ})$ & $E_{\text {out }}(\mathrm{kJ})$ & $E_{d}(\mathrm{~kJ})$ & $E_{b}(\mathrm{~kJ})$ & $\eta$ & $\zeta$ \\
\hline 0.411 & 32.161 & 8.562 & 23.599 & 2.323 & 0.734 & 0.098 \\
1.169 & 39.542 & 9.661 & 29.881 & 2.502 & 0.756 & 0.084 \\
2.141 & 46.971 & 10.618 & 36.354 & 2.586 & 0.774 & 0.071 \\
3.111 & 54.361 & 11.734 & 42.618 & 2.854 & 0.784 & 0.067 \\
3.944 & 61.685 & 13.121 & 48.563 & 3.003 & 0.787 & 0.062 \\
4.795 & 69.006 & 14.275 & 54.731 & 3.163 & 0.793 & 0.058 \\
5.866 & 76.230 & 15.389 & 60.840 & 3.282 & 0.798 & 0.054 \\
6.185 & 83.554 & 16.531 & 67.022 & 3.537 & 0.802 & 0.053 \\
6.525 & 90.777 & 18.117 & 72.660 & 3.697 & 0.800 & 0.051 \\
7.093 & 98.022 & 19.762 & 78.260 & 3.805 & 0.798 & 0.049 \\
\hline
\end{tabular}

when $\mathrm{Fr}$ becomes large.

To further quantify the observed relation between the energy dissipation ratio $\eta$ and $F r$, an inverse correlation between the energy escaping ratio $1-\eta$ and $F r$ is plot in Fig. 15. The energy escaping ratio denotes the ratio of outflow energy $E_{\text {out }}$ to the energy of incoming flow $E_{\text {in }}$. As mentioned earlier, $E_{\text {out }}$ is the sum of kinetic and potential energy of the debris fluid and debris particles escaped from the barrier $\left(E_{k_{-} \text {out }}^{\text {fluid }}, E_{p_{-} \text {out }}^{\text {fluid }}\right.$, $\left.E_{k_{-} \text {out }}^{\text {particle }}, E_{p_{-} \text {out }}^{\text {particle }}\right)$. As demonstrated in Fig. 16 , the energy of outflow is primarily contributed by the debris fluid, as debris particles are effectively blocked (see Fig. 10). $E_{k_{-} \text {out }}^{\text {fluid }} / E_{\text {in }}$ shows a positive correlation with $F r$, reflecting more kinetic energy of the escaped fluid with a large $F r$ and a high velocity of incoming flow. Meanwhile, $E_{p_{-} \text {out }}^{\text {fluid }} / E_{\text {in }}$ is negatively correlated with Fr. Indeed, a large $F r$ and a small flow height reduce the potential energy of the escaped fluid. When $F r$ is smaller $(\lesssim 5.866)$, the decrease of the potential energy of the outflowing fluid $\left(E_{p_{-} \text {out }}^{\text {fluid }} / E_{\text {in }}\right)$ dominates the energy of the escaped debris mass, leading to the initial decreasing trend of the energy escaping ratio (i.e., 1- $\eta$ ) in Fig. 15. After Fr becomes larger, the effect of increasing kinetic energy of the outflowing fluid $\left(E_{k_{-} \text {out }}^{\text {fluid }} / E_{\text {in }}\right.$ ) becomes notable, which balances the reduction in the potential energy of the outflowing fluid $\left(E_{p_{-} \text {out }}^{\text {fluid }} / E_{\text {in }}\right)$ and leads to the stable stage in Fig. 15.

The change of energy absorption ratio $\zeta$ with Froude number $F r$ is shown in Fig. 17. It is interesting to observe a negative correlation between $\zeta$ and $F r$, in contrast with the positive relation between the energy dissipation ratio $\eta$ and Fr in Fig. 15. The reduction of $\zeta$ with Fr indicates that the dissipated energy is less contributed from the absorbed energy by the flexible barrier, whilst the increase of $\eta$ with $F r$ means more energy dissipation with a larger Fr. Given a fixed amount of energy dissipation $\left(E_{\text {in }}-E_{\text {out }}\right)$, the higher the $F r$, the more the energy dissipation by 1) internal friction and collision within the debris mass; 2) interaction between the debris mass and boundaries; 3 ) sliding and collisions among barrier components, and thus the less energy stored/absorbed by a flexible barrier. This confirms a flexible barrier is more efficient to mitigate a debris flow with a higher $\mathrm{Fr}$ until a certain threshold $(\mathrm{Fr} \approx$ 5.866 in this study), in terms of the energy dissipation of the debris flow and the energy absorption of the flexible barrier. Note that the retained debris mass with a large Fr is less (in Fig. 10), indicating the less efficiency of the barrier from the perspective of the blocked debris mass. In practical design, a flexible barrier should be able to resist the impact of a debris flow, and its main tasks are to dissipate the energy of the debris flow and to retain totally or partially the debris mass (Vagnon, 2020). Therefore, the blocked debris mass by the flexible barrier should always be analyzed along with the energy dissipation and the energy absorption. Given the expected magnitude/volume of a debris flow at a specific site, the flow velocity and flow height of the debris flow can be estimated with an assumption of no mitigation measures. The Fr of the flow can be obtained, which changes with the front position along the flow channel. With a prescribed requirement of blocked debris mass, the optimal location of a flexible barrier can be determined according to the relation between the retained debris mass and Fr (as Fig. 10 in this study). Knowing the location of the barrier and the Fr of the debris flow, the energy dissipation ratio can be checked for evaluation of the outflow energy, which may affect the design of downstream barriers if any. Meanwhile, the energy absorption ratio and the force in the barrier need to be examined to avoid barrier failure and to guarantee barrier efficiency.

\section{Discussion}

In this study, an idealised and small-scale setup has been considered to investigate the performance of flexible ring net barriers subjected to the impacts of debris flows with different Froude numbers. For smallscale laboratory experiments and numerical simulations, geometric, kinematic, and dynamic similarities with real-scale debris flows need to 


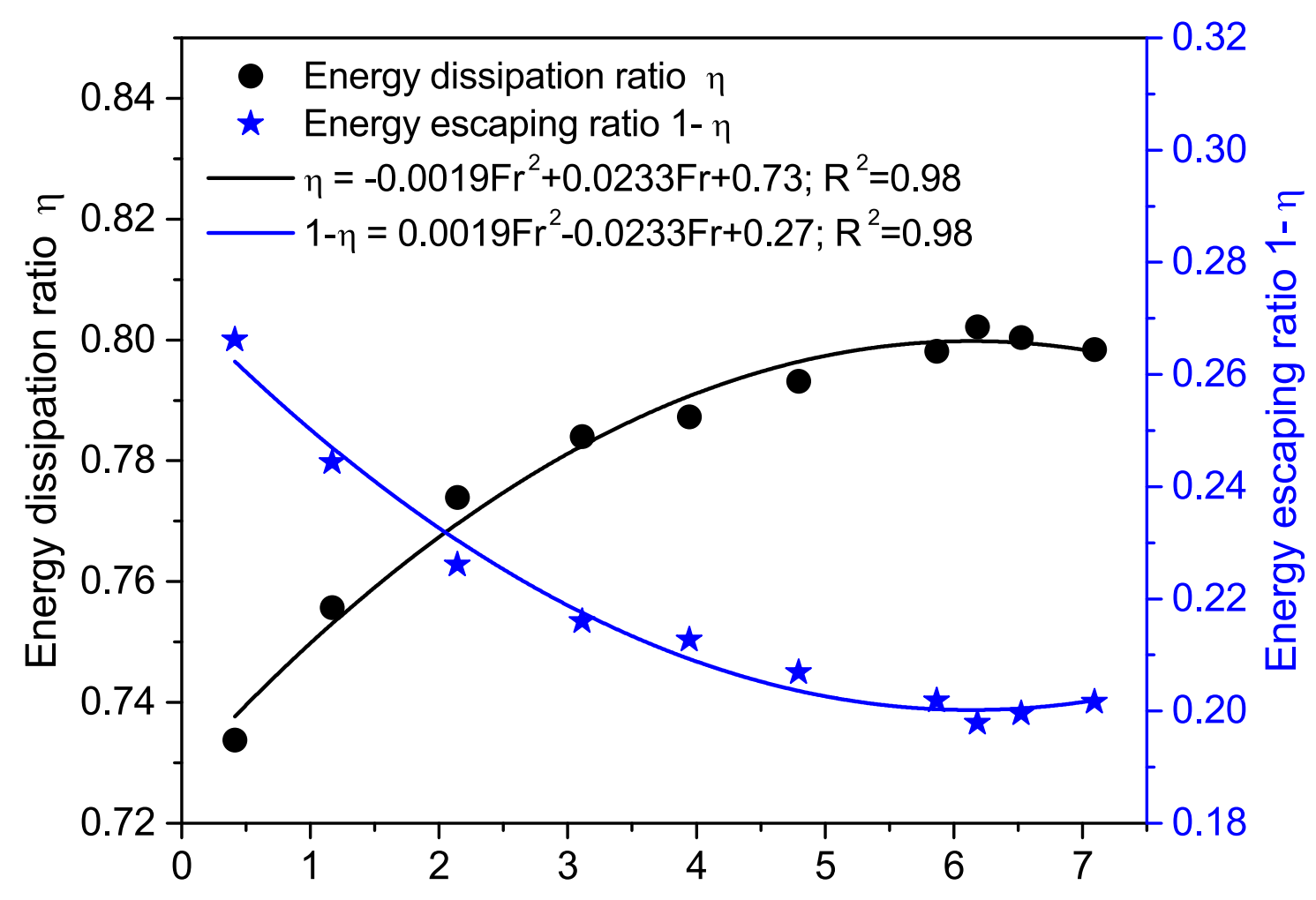

Froude number $\mathrm{Fr}$

Fig. 15. Evolution of energy dissipation ratio and energy escaping ratio with Froude number.

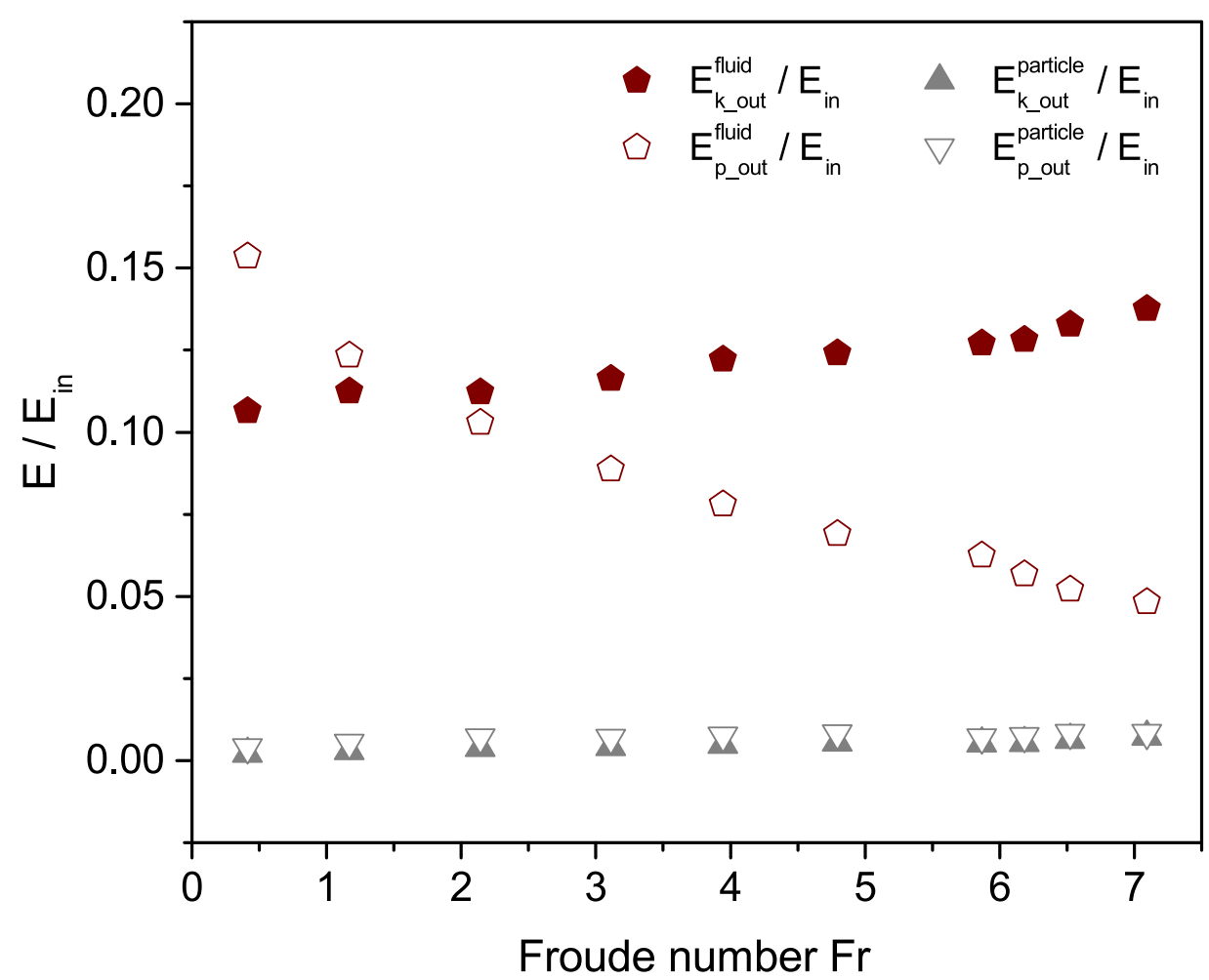

Fig. 16. Change of different components of the outflow energy with Froude number. The components include kinetic and potential energy of the escaped debris fluid and particles ( $\left.E_{k_{-} \text {out }}^{\text {flud }}, E_{p_{-} \text {out }}^{\text {flue }}, E_{k_{-} \text {out }}^{\text {particle }}, E_{p_{-} \text {out }}^{\text {particle }}\right)$. 


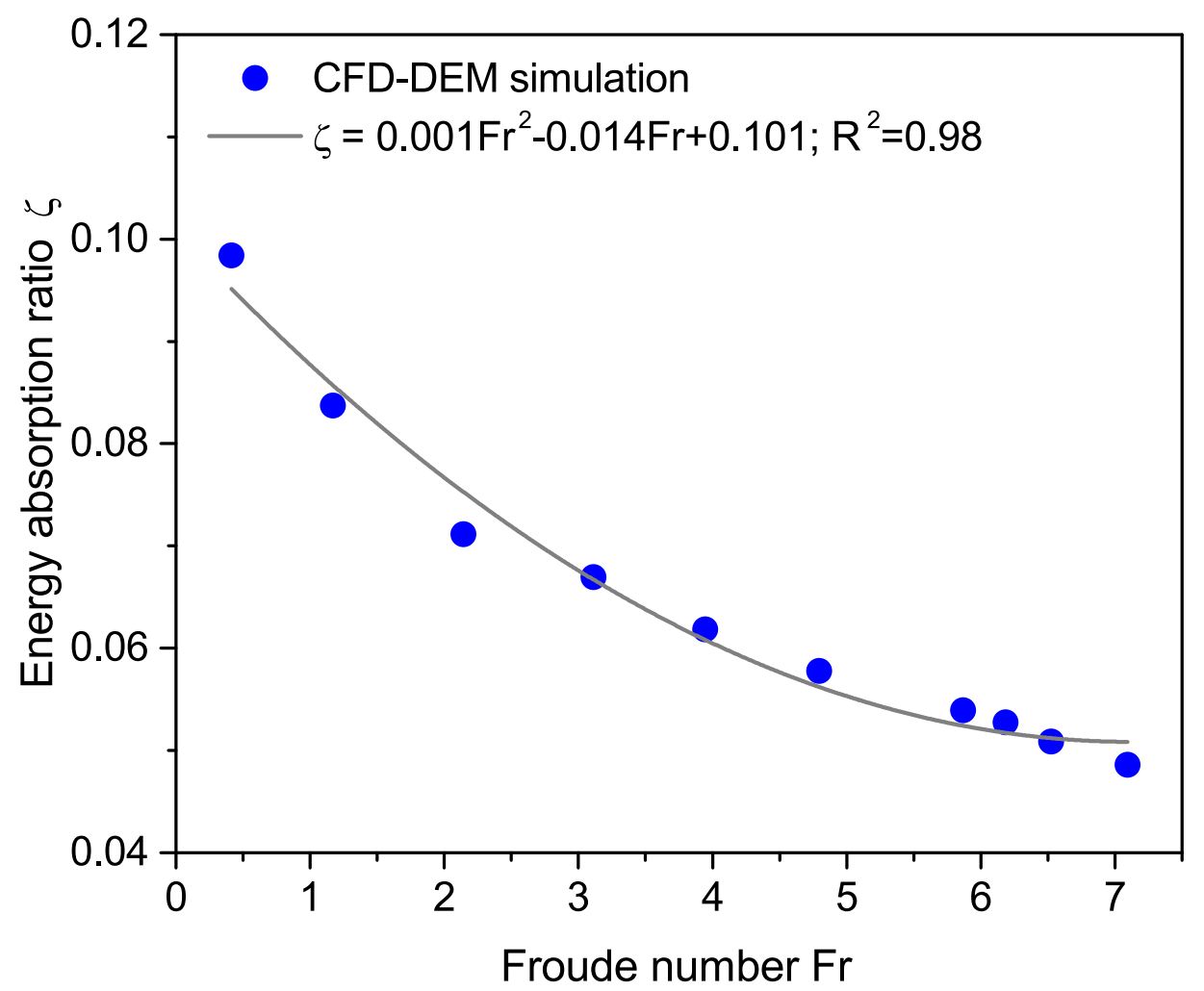

Fig. 17. Evolution of energy absorption ratio with Froude number.

be fully considered. Nevertheless, it is challenging to simultaneously satisfy all the similarities, and a leading similarity is normally selected (Wendeler and Volkwein, 2015). As shown in Section 4.1, this study fulfills the dynamic similarity by applying the Froude number, which has been identified as a key dimensionless parameter governing the impact behavior of debris flow and has been widely adopted in smallscale experiments (Scheidl et al., 2013; Wendeler and Volkwein, 2015; Choi et al., 2015). The absolute values of the results from small-scale studies might not be directly referred to in their corresponding largescale investigations. Hence, the performance of the flexible ring net barrier in Section 4 has been mainly discussed with normalized quantities to be scale free. It is worth noting that although this study applies a small-scale setup for computational efficiency, the coupled CFD-DEM approach can indeed be adopted to model complex real-scale debris flows (Kong et al., 2018). Apart from the scale of the simulations, the idealised setup in this study can be further adjusted from various aspects (e.g. the debris mixture, the flexible barrier, and the channel) to explore more working conditions as discussed below.

A typical debris flow in reality is normally composed of water and a wide range of solid particles from clay to boulders. The magnitude of the particle size may vary from micrometer (e.g. clay and silt) to meter (e.g. boulder). Since the time step of DEM is constrained by the smallest particle in the simulation, it is extremely expensive to simulate a debris particle system with a complete grain size distribution by DEM. As a simplification, a bi-dispersed granular packing has been assumed in our simulations. To study the effect of debris particles, a wider grain size distribution could be further considered. In addition, a debris flow can be modeled by the CFD-DEM approach with consideration of a complete grain size distribution, by taking fine particles into the fluid phase solved with CFD. In doing so, only large particles need to be modeled with DEM, which can greatly reduce the computational cost. Meanwhile, instead of water, a mud flow composed of water and fine particles needs to be solved with CFD.

Depending on the channel geometry, practical design may adopt a single-span flexible barrier or a multi-span barrier system. If a channel is narrow ( $\leqslant 12 \sim 15 \mathrm{~m}$ ), a single-span barrier with no posts is sufficient (Wendeler et al., 2007). Otherwise, a multi-span barrier with fixing posts may be considered. In addition to the single-span barrier focused in this study, the CFD-DEM method can also tackle multi-span flexible ring net barriers impacted by debris flows, in further consideration of posts. Based on the field observation by Kwan et al. (2014), posts can be damaged with different possible reasons, including direct impact of debris mass, connection between the post and the ring net, and connection between the post and its foundation. All these different causes can be readily investigated by designing different scenarios in CFD-DEM simulations, and will be considered in the future.

A rectangular chute has been assumed as the flow channel in this study. The flow dynamics may be notably affected by the topographic features of the flow channel (Han et al., 2014). To naturally constrain the path of debris flow, a more complex terrain from laboratory experiments (Iverson et al., 2004) and real terrain from the field (AECOM, 2012) can be implemented to the CFD-DEM modeling (Kong et al., 2018). Moreover, erosion of debris flow can be captured by considering an erodible bed modeled with bonded particles (Li, 2018; Kong et al., 2018). Despite of the idealized conditions considered in this study, we have demonstrated that the proposed numerical approach serves as a promising tool for future study of debris flow impacts on flexible ring net barriers. Nevertheless, to provide rational analyses for practical design of a flexible ring net barrier, the modeling of all barrier components need to be further carefully calibrated and verified with laboratory experiments and on-site tests.

\section{Conclusions}

This study has presented a unified CFD-DEM framework to investigate the dynamic impact of debris flows on flexible ring net barriers. The debris flow is modeled as a solid-fluid mixture, where the fluid and solid phases are simulated with the CFD and the DEM, respectively. The flexible ring net barrier, typically composed of cables, rings and energy dissipators, is modeled by the DEM with bonded particles. The cables 
and rings are assumed to have constant stiffness. The energy dissipators are modeled with a tri-linear or bi-linear law to capture their changing stiffness depending on the loading level. The physical contacts of the barrier components, like frictional sliding and collisions, are controlled by the particle property. The responses of the barrier, including deformation, are governed by the bond properties. The proposed computational method has been proved as a reliable tool according to the simulations of quasi-static loading on single barrier components and dynamic loading on an entire flexible barrier. The force-displacement responses of a ring and an energy dissipator from the simulations agree well with the experimental data from the literatures. The displacement and energy of the rock in the simulation of rockfall impact on a flexible barrier are consistent with the measurement data as well. Moreover, the well-known curtain effect of a ring net has been captured in the simulation of dynamic rockfall impact, by virtue of the allowed sliding of the rings along the attached cables

The performance of a flexible ring net barrier under debris flow impacts has been further examined, considering debris flows with different Froude numbers. It is found the blocked debris mass by the barrier is negatively correlated with Froude number. Two patterns of force distribution within a flexible barrier have been identified, one with vertical strong force chains, and the other with horizontal ones. Both the peak total forces sustained by the cables and by the entire barrier have been evaluated. It is interesting that the peak total force sustained by the most critical cable seems to be negatively correlated with Froude number, in contrast with the positive correlation between the peak total force sustained by the entire barrier and the Froude number. This finding highlights the importance of analyzing the force distribution within a barrier and the force in key barrier components, in addition to the overall force sustained by the entire barrier. Furthermore, the energy dissipation ratio and the energy absorption ratio are suggested for the evaluation of barrier efficiency. In terms of dissipated energy of the debris flow and absorbed energy by the flexible barrier, a higher Froude number leads to a more efficient barrier, which differs from the barrier efficiency examined based on retained debris mass. An efficient barrier from the perspective of energy might not be always satisfactory from the view of blocked debris mass. Therefore, the analysis of barrier capability and efficiency requires a combination of comprehensive investigations on the retained debris mass, the force in the barrier, the energy dissipation of the debris flow and the energy absorption by the barrier. The proposed unified framework enables such a possibility for future flexible barrier design.

\section{CRediT authorship contribution statement}

Xingyue Li: Conceptualization, Methodology, Software, Writing original draft. Jidong Zhao: Conceptualization, Supervision, Writing review \& editing. Julian S.H. Kwan: Methodology, Writing - review \& editing.

\section{Declaration of Competing Interest}

The authors declare that they have no known competing financial interests or personal relationships that could have appeared to influence the work reported in this paper.

\section{Acknowledgments}

The research in this study was financially supported by the National Natural Science Foundation of China (via Project \#11972030) and University Grants Council of Hong Kong by a General Research Fund project (GRF \#16205418), a Theme-based Research Scheme project (TBRS \#T22-603/15N) and a Collaborative Research Fund project (CRF \#C6012-15G). This paper is published with the permission of the Head of Geotechnical Engineering Office and the Director of Civil Engineering and Development, Government of the Hong Kong SAR, China.

\section{References}

AECOM, 2012. Detailed study of the 7 June 2008 landslides on the hillside above Yu Tung Road, Tung Chung, Geotechnical Engineering Office, HKSAR, GEO Report 271.

Albaba, A., Lambert, S., Kneib, F., Chareyre, B., Nicot, F., 2017. DEM modeling of a flexible barrier impacted by a dry granular flow. Rock Mech. Rock Eng. 50, 3029-3048.

Bertrand, D., Nicot, F., Gotteland, P., Lambert, S., 2008. Discrete element method (DEM) numerical modeling of double-twisted hexagonal mesh. Can. Geotech. J. 45, 1104-1117.

Bertrand, D., Trad, A., Limam, A., Silvani, C., 2012. Full-scale dynamic analysis of an innovative rockfall fence under impact using the discrete element method: from the local scale to the structure scale. Rock Mech. Rock Eng. 45, 885-900.

Brighenti, R., Segalini, A., Ferrero, A.M., 2013. Debris flow hazard mitigation: a simplified analytical model for the design of flexible barriers. Comput. Geotech. 54, $1-15$.

Bugnion, L., Bötticher, A., Wendeler, C., 2012. Large scale field testing of hillslope debris flows resulting in the design of flexible protection barriers. In: Proceedings of 12th Interpraevent Grenoble/France.

Bugnion, L., Wendeler, C., 2010. Shallow landslide full-scale experiments in combination with testing of a flexible barrier. WIT Trans. Eng. Sci. 67, 161-173.

Buzzi, O., Spadari, M., Giacomini, A., Fityus, S., Sloan, S., 2013. Experimental testing of rockfall barriers designed for the low range of impact energy. Rock Mech. Rock Eng. 46, 701-712.

Castanon-Jano, L., Blanco-Fernandez, E., Castro-Fresno, D., Ballester-Muñoz, F., 2017. Energy dissipating devices in falling rock protection barriers. Rock Mech. Rock Eng. 50, 603-619.

Chan, S., Zhou, Z., Liu, Y., 2012. Numerical analysis and design of flexible barriers allowing for sliding nodes and large deflection effects. In: Proceedings of the One Day Seminar on Natural Terrain Hazards Mitigation Measures, pp. 29-43.

Choi, C., Ng, C.W.W., Law, R.P., Song, D., Kwan, J., Ho, K., 2015. Computational investigation of baffle configuration on impedance of channelized debris flow. Can. Geotech. J. 52, 182-197.

Coulibaly, J.B., Chanut, M.A., Lambert, S., Nicot, F., 2019. Toward a generic computational approach for flexible rockfall barrier modeling. Rock Mech. Rock Eng. 52, 4475-4496.

Dang, T.S., Meschke, G., 2014. An ALE-PFEM method for the numerical simulation of two-phase mixture flow. Comput. Methods Appl. Mech. Eng. 278, 599-620.

Derakhshani, S.M., Schott, D.L., Lodewijks, G., 2016. Calibrating the microscopic properties of quartz sand with coupled CFD-DEM framework. Eng. Comput. 33, 1141-1160.

Gao, Z., Al-Budairi, H., Steel, A., 2018. Experimental testing of low-energy rockfall catch fence meshes. J. Rock Mech. Geotech. Eng. 10, 798-804.

Giacomini, A., Thoeni, K., Lambert, C., Booth, S., Sloan, S., 2012. Experimental study on rockfall drapery systems for open pit highwalls. Int. J. Rock Mech. Min. Sci. 56, $171-181$.

Goniva, C., Kloss, C., Hager, A., Pirker, S., 2010. An open source CFD-DEM perspective. In: Proceedings of OpenFOAM Workshop, Göteborg, pp. 22-24.

Grassl, H., Bartelt, P., Volkwein, A., Wartmann, S., 2003. Experimental and numerical modeling of highly flexible rockfall protection barriers. In: Proceedings of 12th Panamerican Conference on Soil Mechanics and Geotechnical Engineering, Cambridge, Massachusetts, USA, p. 2594.

Han, Z., Chen, G., Li, Y., Xu, L., Zheng, L., Zhang, Y., 2014. A new approach for analyzing the velocity distribution of debris flows at typical cross-sections. Nat. Hazards 74, 2053-2070.

Hu, K., Wei, F., Li, Y., 2011. Real-time measurement and preliminary analysis of debrisflow impact force at Jiangjia Ravine, China. Earth Surf. Proc. Land. 36, 1268-1278.

Hübl, J., Suda, J., Proske, D., Kaitna, R., Scheidl, C., 2009. Debris flow impact estimation. In: Proceedings of the 11th International Symposium on Water Management and Hydraulic Engineering, Ohrid, Macedonia, pp. 1-5.

Huo, M., Zhou, J., Yang, X., Zhou, H., 2017. Effects of a flexible net barrier on the dynamic behaviours and interception of debris flows in mountainous areas. J. Mount. Sci. 14, 1903-1918.

Huo, M., Zhou, J., Yang, X., Zhou, H., 2018. Dynamical evolution properties of debris flows controlled by different mesh-sized flexible net barriers. Arab. J. Geosci. 11, 465.

Iverson, R.M., Logan, M., Denlinger, R.P., 2004. Granular avalanches across irregular three-dimensional terrain: 2. Experimental tests. J. Geophys. Res.: Earth Surface 109.

Kong, Y., Zhao, J., Li, X., Kwan, J., Sze, E., 2018. Coupled CFD/DEM modeling of multiphase debris flow over a natural erodible terrain: the Yu Tung road case. In: Second JTC1 Workshop on Triggering and Propagation of Rapid Flow-like Landslides.

Koo, R.C., Kwan, J.S., Lam, C., Ng, C.W., Yiu, J., Choi, C.E., Ng, A.K., Ho, K.K., Pun, W., 2017. Dynamic response of flexible rockfall barriers under different loading geometries. Landslides 14, 905-916.

Kwan, J., 2012. Supplementary technical guidance on design of rigid debris-resisting barriers. Geotechnical Engineering Office, HKSAR, GEO Report 270.

Kwan, J., Chan, S., Cheuk, J., Koo, R., 2014. A case study on an open hillside landslide impacting on a flexible rockfall barrier at Jordan Valley, Hong Kong. Landslides 11, 1037-1050.

Kwan, J.S., Sze, E.H., Lam, C., 2018. Finite element analysis for rockfall and debris flow mitigation works. Can. Geotech. J. 1-26.

Lau, A., Anson, M., 2006. Effect of high temperatures on high performance steel fibre reinforced concrete. Cem. Concr. Res. 36, 1698-1707. 
Leonardi, A., Wittel, F.K., Mendoza, M., Vetter, R., Herrmann, H.J., 2016. Particle-fluid-structure interaction for debris flow impact on flexible barriers. Comput.-Aided Civ. Infrastruct. Eng. 31, 323-333.

Li, X., 2018. Computational modelling of debris flows and their interaction with resisting barriers based on a coupled CFD-DEM approach. Ph.D. thesis. The Hong Kong University of Science and Technology.

Li, X., Zhao, J., 2018. Dam-break of mixtures consisting of non-newtonian liquids and granular particles. Powder Technol. 338, 493-505.

Li, X., Zhao, J., 2018. A unified CFD-DEM approach for modeling of debris flow impacts on flexible barriers. Int. J. Numer. Anal. Meth. Geomech. 42, 1643-1670.

Li, X., Zhao, J., Soga, K., 2020. A new physically based impact model for debris flow. Géotechnique 1-12.

Mao, J., Zhao, L., Di, Y., Liu, X., Xu, W., 2020. A resolved CFD-DEM approach for the simulation of landslides and impulse waves. Comput. Methods Appl. Mech. Eng. 359, 112750.

Marchelli, M., De Biagi, V., Peila, D., 2019. A quick-assessment procedure to evaluate the degree of conservation of rockfall drapery meshes. Frattura ed Integrità Strutturale 13, 437-450.

Ng, C., Song, D., Choi, C., Liu, L., Kwan, J., Koo, R., Pun, W., 2016. Impact mechanisms of granular and viscous flows on rigid and flexible barriers. Can. Geotech. J. 54, 188-206.

Nicot, F., Cambou, B., Mazzoleni, G., 2001. Design of rockfall restraining nets from a discrete element modelling. Rock Mech. Rock Eng. 34, 99-118.

Potyondy, D.O., Cundall, P., 2004. A bonded-particle model for rock. Int. J. Rock Mech. Min. Sci. 41, 1329-1364.

Rhebergen, S., Bokhove, O., van der Vegt, J.J., 2009. Discontinuous Galerkin finite element method for shallow two-phase flows. Comput. Methods Appl. Mech. Eng. 198, 819-830.

Scheidl, C., Chiari, M., Kaitna, R., Müllegger, M., Krawtschuk, A., Zimmermann, T., Proske, D., 2013. Analysing debris-flow impact models, based on a small scale modelling approach. Surv. Geophys. 34, 121-140.

Shan, T., Zhao, J., 2014. A coupled CFD-DEM analysis of granular flow impacting on a water reservoir. Acta Mech. 225, 2449-2470.

Smuts, E.M., Deglon, D.A., Meyer, C.J., 2012. Methodology for CFD-DEM modelling of particulate suspension rheology. In: Proceedings of the Ninth International Conference on CFD in the Minerals and Process Industries CSIRO, Melbourne, Australia.
Song, D., Zhou, G.G., Xu, M., Choi, C., Li, S., Zheng, Y., 2019. Quantitative analysis of debris-flow flexible barrier capacity from momentum and energy perspectives. Eng. Geol. 251, 81-92.

Tao, H., Tao, J., 2017. Quantitative analysis of piping erosion micro-mechanisms with coupled CFD and DEM method. Acta Geotech. 12, 573-592.

Thoeni, K., Giacomini, A., Lambert, C., Sloan, S.W., Carter, J.P., 2014. A 3D discrete element modelling approach for rockfall analysis with drapery systems. Int. J. Rock Mech. Min. Sci. 68, 107-119.

Vagnon, F., 2020. Design of active debris flow mitigation measures: a comprehensive analysis of existing impact models. Landslides 17, 313-333.

Volk, A., Ghia, U., Liu, G.R., 2018. Assessment of CFD-DEM solution error against computational cell size for flows through a fixed-bed of binary-sized particles. Powder Technol. 325, 519-529.

Volkwein, A., 2004. Numerische Simulation von Flexiblen Steinschlagschutzsystemen. Ph.D. thesis, Eidgenössische Technische Hochschule Zürich.

Volkwein, A., 2005. Numerical simulation of flexible rockfall protection systems. Compu. Civ. Eng. 1-11.

Volkwein, A., Wendeler, C., Stieglitz, L., Lauber, G., 2015. New approach for flexible debris flow barriers. In: IABSE Symposium Report, pp. 1-7.

Wendeler, C., Budimir, V., Denk, M., 2018a. Debris flow protection with flexible ring net barriers-10 years of experience. ce/papers 2, 1039-1044.

Wendeler, C., McArdell, B., Volkwein, A., Denk, M., Gröner, E., 2008. Debris flow mitigation with flexible ring net barriers-field tests and case studies. WIT Trans. Eng. Sci. 60, 23-31.

Wendeler, C., Volkwein, A., 2015. Laboratory tests for the optimization of mesh size for flexible debris-flow barriers. Nat. Hazards Earth Syst. Sci. 15.

Wendeler, C., Volkwein, A., McArdell, B.W., Bartelt, P., 2018b. Load model for designing flexible steel barriers for debris flow mitigation. Can. Geotech. J. 56, 893-910.

Wendeler, C., Volkwein, A., Roth, A., Denk, M., Wartmann, S., 2007. Field measurements and numerical modelling of flexible debris flow barriers. Debris-Flow Hazards Mitigation Mechanics, Prediction and Assessment. Millpress, Rotterdam, 681-687.

Yiu, J., Huang, Y., Pappin, J., Sturt, R., Kwan, J., Ho, K., 2012. Landslide mobility and flexible barrier numerical modeling using LS-DYNA. In: 12th International LS-DYNA Users Conference.

Zhao, J., Shan, T., 2013. Coupled CFD-DEM simulation of fluid-particle interaction in geomechanics. Powder Technol. 239, 248-258. 